\title{
Characterization of Endogenous Sodium Channels in the ND7-23 Neuroblastoma Cell Line: Implications for Use as a Heterologous Ion Channel Expression System Suitable for Automated Patch Clamp Screening
}

\author{
Marc Rogers, ${ }^{1, *}$ Nace Zidar, ${ }^{2}$ Danijel Kikelj, and Robert W. Kirby ${ }^{1, *}$ \\ ${ }^{7}$ Xention Limited, Cambridge, United Kingdom. \\ ${ }^{2}$ Faculty of Pharmacy, University of Ljubljana, Ljubljana, Slovenia. \\ *Present address: Metrion Biosciences Ltd., Cambridge, \\ United Kingdom.
}

\section{ABSTRACT}

The rodent neuroblastoma cell line, ND7-23, is used to express voltage-dependent sodium (Nav) and other neuronal ion channels resistant to heterologous expression in Chinese hamster ovary (CHO) or human embryonic kidney (HEK) cells. Their advantage is that they provide endogenous factors and signaling pathways to promote ion channel peptide folding, expression, and function at the cell surface and are also amenable to automated patch clamping. However, ND7-23 cells exhibit endogenous tetrodotoxin (TTX)-sensitive Nav currents, and molecular profiling has revealed the presence of Nav1.2, Nav1.3, Nav1.6, and Nav1.7 transcripts, but no study has determined which subtypes contribute to functional channels at the cell surface. We profiled the repertoire of functional Nav channels endogenously expressed in ND7-23 cells using the QPatch automated patch clamp platform and selective toxins and small molecules. The potency and subtype selectivity of the ligands (Icagen compound 68 from patent US-20060025415-A120060202, 4,9 anhydro $\Pi X$, and Protoxin-II) were established in human Nav1.3, Nav1.6, and Nav1.7 channel cell lines before application of selective concentrations to ND7-23 cells. Our data confirm previous studies that $>97 \%$ of macroscopic Nav current in ND7-23 cells is carried by TTX-sensitive channels ( $300 \mathrm{nM} \Pi \mathrm{TX}$ ) and that Nav1.7 is the predominant channel contributing to this response (65\% of peak inward current), followed by Nav1.6 $(\sim 20 \%)$ and negligible Nav1.3 currents ( $\sim 2 \%)$. In addition, our data are the first to assess the Nav1.6 potency (50\% inhibitory concentration $\left[{ }^{1} \mathrm{C}_{50}\right]$ of $33 \mathrm{nM}$ ) and selectivity (50-fold over Nav1.7) of 4,9 anhydro TTX in human Nav channels expressed in mammalian cells, confirming previous studies of rodent Nav channels expressed in oocytes and HEK cells.

\section{INTRODUCTION}

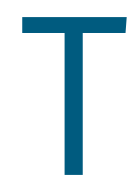

he sodium channel (Nav) gene family is classified into tetrodotoxin-sensitive (TTX-S; Nav1.1, Nav1.2, Nav1.3, Nav1.4, Nav1.6, and Nav1.7) and TTX-resistant (TTX-R) channels (Nav1.5, Nav1.8, and Nav1.9), each of which is associated with specific therapeutic indications based on their expression pattern, function, and genetic mutations (reviewed in Refs. ${ }^{1-3}$ ). Neuronal voltage-gated sodium channels are important drug discovery targets for pain (Nav1.3, Nav1.7, Nav1.8, Nav1.9), epilepsy (Nav1.1, Nav1.2), and multiple sclerosis (Nav1.6). ${ }^{4,5}$ High-throughput screening, hit validation, lead optimization, and gene family selectivity now all largely rely on heterologous expression of specific Nav ion channel subunits in a limited set of mammalian cell backgrounds amenable to cellbased assay and automated patch clamp (APC) electrophysiology platforms. For example, most TTX-sensitive Nav channels express well in human embryonic kidney (HEK) cells, ${ }^{6,7}$ but it is noteworthy that HEK cells also exhibit significant levels (100$500 \mathrm{pA}$ ) of endogenous TTX-S and TTX-R Nav currents and express Nav1.2, Nav1.3, Nav1.7, and Nav1.5 subunits. ${ }^{89}$ In contrast, mutant Nav1.6 channels associated with ataxia and epilepsy $^{10,11}$ and TTX-resistant Nav1.8 and Nav1.9 channels

(c) Marc Rogers et al., 2016; Published by Mary Ann Liebert, Inc. This Open Access article is distributed under the terms of the Creative Commons Attribution Noncommercial License (http://creativecommons.org/licenses/by-nc/4.0/) which permits any noncommercial use, distribution, and reproduction in any medium, provided the original author(s) and the source are credited. 
implicated in neuropathic, inflammatory, and visceral pain have proven resistant to heterologous expression in fibroblast-like Chinese hamster ovary (CHO) or HEK cells. ${ }^{7,12-15}$ Several groups have therefore turned to immortalized neuroblastoma cell lines that contain a more diverse and appropriate set of accessory proteins, ${ }^{16}$ successfully expressing mutant Nav1.6 channels in rodent ND7-23 neuroblastoma cells ${ }^{11}$ and Nav1.8 channels in human SH-SY5Y ${ }^{17}$ and rodent ND7-23 cell lines ${ }^{7,18-22}$ and more recently the recalcitrant hNav1.9 subunit in ND7-23 cells. ${ }^{23-25}$

Although the heterologous expression of Nav1.6 mutant and TTX-resistant Nav channels is higher in neuroblastoma cell lines compared with HEK cells, both of these cell types exhibit a background of endogenous Nav channel activity. This can reduce the signal window as well as compromise the fidelity of drug discovery assays designed to detect subtype selective Nav ligands with improved therapeutic and side effect profiles. ${ }^{3,4}$ It is therefore important that both the level of background expression and mix of Nav ion channel subtypes are determined in the various cell lines being used as hosts for heterologous expression of human Nav channels to ensure reliable ion channel drug screening. There are a variety of subtype-selective Nav antagonists available that originated from such drug discovery efforts, which can be used to define Nav1.x expression profiles in native systems. In this study, we used an Nav1.3-selective small molecule patented by Icagen, ${ }^{26}$ and the Nav1.7-selective tarantula spider toxin Protoxin-II that was used by Merck \&t Co., Inc., in their pain drug discovery program, ${ }^{27}$ which derives its selectivity through binding to divergent voltage sensor domains on Nav1.x channels. ${ }^{28}$ Finally, we used the naturally occurring TTX metabolite 4,9 anhydro TTX ${ }^{29}$ that was first shown to be selective for rodent Nav1.6 channels expressed in Xenopus oocytes $^{30}$ to define the proportion of Nav1.x channels in ND723 neuroblastoma cells.

The ND7-23 immortalized rat dorsal root ganglion (DRG)mouse N18Tg2 neuroblastoma hybrid cell line ${ }^{31}$ has shown great utility for transient and stable expression of exogenous ion channels and receptors in a native milieu, yielding useful fluorescence and electrophysiology assays for academic and drug discovery applications. Given the frequent use of ND7-23 neuroblastoma cells to express TTX-resistant Nav channels and their suitability for APC electrophysiology screening assays, there is a lack of consensus in the literature on the endogenous Nav subunits expressed in this cell line. Several groups have used molecular techniques to identify the presence of mRNA or protein for $\beta 1$ and $\beta 3$ accessory subunits ${ }^{7}$ and Nav1.2, Nav1.3, Nav1.6, and Nav1.7 pore-forming $\alpha$ subunits in ND7-23 cells. ${ }^{32,33}$ However, none of these studies confirmed the contribution of these particular Nav channel subtypes to functional macroscopic currents. Similarly, patch clamp studies of TTXresistant Nav channels heterologously expressed in ND7-23 cells did not profile the underlying endogenous Nav currents other than to establish that they were completely blocked by TTX. ${ }^{7,18,20,21,23,32}$ We therefore undertook to define the repertoire of endogenous Nav1.x channels functionally expressed on ND7-23 neuroblastoma cells using subtype-selective pharmacological ligands.

\section{MATERIALS AND METHODS}

Compounds

The Icagen Nav1.3 compound 68 was synthesized according to Gonzalez et al. ${ }^{26}$ and details are given below (Fig. 1). 4,9 Anhydro TTX was synthesized commercially and kindly shared by Mr. Ari Alexandrou (Pfizer-Neusentis). Synthetic ProtoxinII was purchased from Peptanova GmbH. All remaining physiological salts and reagents were obtained from commercial suppliers (Sigma-Aldrich and Calbiochem).

\section{Synthesis of Icagen Nav1.3 Compound 68}

Chemicals were obtained from Acros Organics and SigmaAldrich and used without further purification. Analytical thinlayer chromatography was performed on silica gel Merck 60 F254 plates $(0.25 \mathrm{~mm})$, using visualization with ultraviolet light and spray reagents. High-performance liquid chromatography (HPLC) analyses were performed on an Agilent Technologies 1100 instrument (Agilent Technologies) with a G1365B UV-Vis detector, a G1316A thermostat, and a G1313A autosampler using a Phenomenex Luna $5 \mu \mathrm{m}$ C18 column $(4.6 \times 150$ or $4.6 \times 250 \mathrm{~mm}$; Phenomenex) and a flow rate of $1.0 \mathrm{~mL} / \mathrm{min}$. The eluent consisted of trifluoroacetic acid (TFA; $0.1 \%$ in water) as solvent A and acetonitrile as solvent B. Melting points were determined on a Reichert hot-stage microscope and are uncorrected. ${ }^{1} \mathrm{H}$ and ${ }^{13} \mathrm{C}$ NMR (nuclear magnetic resonance) spectra were recorded at 400 and $100 \mathrm{MHz}$, respectively, on a Bruker AVANCE III 400 spectrometer (Bruker Corporation) in dimethyl sulfoxide (DMSO)- $\mathrm{d}_{6}$, with TMS as the internal

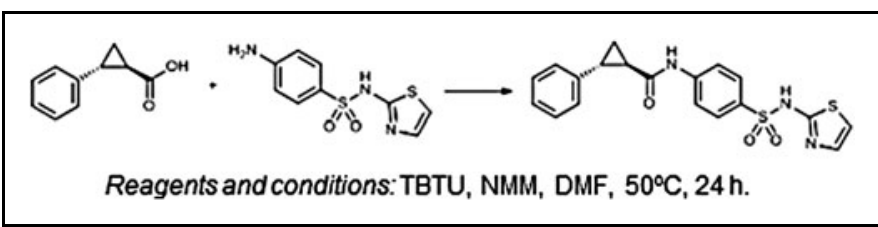

Fig. 1. Synthetic route for Icagen compound 68. Schematic showing starting materials and experimental conditions leading to synthesis of Icagen patent compound $68(1 R, 2 R)-2$-phenyl- $N-(4-(N-$ (thiazol-2-yl)sulfamoyl)phenyl)cyclopropane-1-carboxamide. DMF, dimethyl formamide; NMM, $N$-methylmorpholine, TBTU, $N, N, N^{\prime}, N^{\prime}$ tetramethyl-O-(benzotriazol-1-yl)uronium tetrafluoroborate. 
standard. Infrared (IR) spectra were recorded on a PerkinElmer Spectrum BX FT-IR spectrometer (PerkinElmer, Inc.) or Thermo Nicolet Nexus 470 ESP FT-IR spectrometer (Thermo Fisher Scientific). Mass spectra were obtained using an Advion Expression CMS mass spectrometer (Advion).

The synthetic procedure for $(1 R, 2 R)-2$-phenyl- $N-(4-(N-$ (thiazol-2-yl)sulfamoyl)phenyl)cyclopropane-1-carboxamide (Fig. 1) is as follows. To the solution of $(1 R, 2 R)-2$ phenylcyclopropane-1-carboxylic acid (100 $\mathrm{mg}, 0.617 \mathrm{mmol}$ ) and TBTU $(238 \mathrm{mg}, 0.740 \mathrm{mmol})$ in DMF $(3 \mathrm{~mL}), \quad N$ methylmorpholine $(203 \mu \mathrm{L}, 1.85 \mathrm{mmol})$ was added, and the mixture was stirred at room temperature for $0.5 \mathrm{~h}$. After that 4-amino- $N$-(thiazol-2-yl)benzenesulfonamide ( $157 \mathrm{mg}, 0.617$ $\mathrm{mmol}$ ) was added and the mixture was stirred at $50^{\circ} \mathrm{C}$ overnight. To the reaction mixture, ethyl acetate $(30 \mathrm{~mL})$ was added and the organic phase was washed with water $(2 \times 10 \mathrm{~mL}), 10 \%$ citric acid $(2 \times 10 \mathrm{~mL}), 1 \mathrm{M} \mathrm{HCl}(3 \times 10 \mathrm{~mL})$, and brine $(2 \times 10 \mathrm{~mL})$, dried over $\mathrm{Na}_{2} \mathrm{SO}_{4}$, and evaporated under reduced pressure. To the residue, ether $(20 \mathrm{~mL})$ was added, the obtained suspension was sonicated, the undissolved solid was filtered off, washed with ether $(10 \mathrm{~mL})$, and dried to obtain $(1 R, 2 R)-2-$ phenyl- $N$-(4-( $N$-(thiazol-2-yl)sulfamoyl)phenyl)cyclopropane1-carboxamide as a pale yellow solid (142 mg); yield was 58\% and melting point was $191^{\circ} \mathrm{C}-195^{\circ} \mathrm{C}$. Spectra are as follows: IR (ATR) $v=3245,3103,3057,2899,1652,1593,1566,1519$, $1406,1313,1282,1254,1193,1138,1085,931,932 \mathrm{~cm}^{-1} ;{ }^{1} \mathrm{H}$ NMR (400 MHz, DMSO-d $\left.{ }_{6}\right) \delta 1.39-1.44(\mathrm{~m}, 1 \mathrm{H}, \mathrm{CH}), 1.49-1.54$ (m, 1H, CH), 1.92-2.00 (m, 1H, CH), 2.07-2.12 (m, 1H, CH), 6.82 (d, $1 \mathrm{H}, \mathrm{J}=4.8 \mathrm{~Hz}$, thiazole-H), 7.18-7.32 (m, 6H, Ar-H), 7.73 (s, $4 \mathrm{H}, \mathrm{Ar}-\mathrm{H}), 10.60$ (s, $1 \mathrm{H}, \mathrm{CONH}), 12.69\left(\mathrm{~s}, 1 \mathrm{H}, \mathrm{SO}_{2} \mathrm{NH}\right) ;{ }^{13} \mathrm{C} \mathrm{NMR}$ $\left(100 \mathrm{MHz}, \mathrm{DMSO}-\mathrm{d}_{6}\right) \delta 15.85,25.32,26.80,108.05,118.37$, 124.35, 125.89, 126.18, 126.97, 128.36, 136.12, 140.55, 142.27, 168.67, 170.39. MS (ESI) m/z (\%)=397.87 ([M-H]-). HPLC was carried out using a Phenomenex Luna $5 \mu \mathrm{m}$ C18 column $(4.6 \times 150 \mathrm{~mm})$; mobile phase: $30 \%-90 \%$ of acetonitrile in TFA $(0.1 \%)$ in $16 \mathrm{~min}, 90 \%$ acetonitrile to $20 \mathrm{~min}$; flow rate $1.0 \mathrm{~mL} /$ min; injection volume: $10 \mu \mathrm{L}$; retention time: $8.475 \min (98.7 \%$ at $280 \mathrm{~nm}$ ).

\section{Cell Culture}

CHO and HEK heterologous cell lines stably expressing hNav1.3 (CYL3003), hNav1.6 (CYL3010), and hNav1.7 channels (CYL3011) were grown according to the manufacturer's instructions (Cytomyx/Millipore). All of the Nav1.x cell lines express the $\alpha$ pore-forming subunit under a single selection agent, with the Nav1.6 and Nav1.7 cell lines presumably interacting with endogenous $\beta 1$ subunits in HEK cells. ${ }^{6,7}$ Routine passaging every 2-3 days using agents such as divalent-free phosphate-buffered saline and Accutase ${ }^{\circledR}$ (ThermoFisher) and growth in suitable tissue culture-treated flasks (Corning ${ }^{(\mathbb{B}}$ ) ensure that cells are maintained in log-phase growth and do not form islands or exceed 75\% confluency, critical parameters for automated patch clamping. ND7-23 cells were obtained from European Collection of Authenticated Cell Cultures and grown according to guidelines to maintain uniform confluency and morphology; cells were not differentiated. ND7-23 cells were passaged in a similar manner to heterologous Nav1.x cells lines to ensure optimum patchability (gigaseal and whole-cell formation, and seal quality) on the QPatch APC platform.

\section{APC Electrophysiology}

All of the Nav channel electrophysiology experiments in this study were carried out on the QPatch APC system (Sophion Bioscience A/S), which we have used to make high-fidelity Nav1.x recordings at Xention since 2006. The QPatch is a silicon chip-based planar patch clamp device allowing for up to 48 parallel recordings during one experimental run. Cells are added to each well on the QPlate chip and drawn by suction onto a small aperture to obtain a gigaohm seal (>100 M $\Omega$ ) between the cell membrane and treated silicon surface, after which a whole-cell recording is achieved by applying a combination of suction and voltage pulses to break through the cell membrane and allow perfusion of the cell interior. Most of our experiments employed single-hole QPlates (2.5 $\mathrm{M} \Omega$ resistance), but 10-hole X-Plates were employed for heterologous hNav1.3 recordings to give improved current amplitude and more consistent stability. The QPatch platform and silicon chip QPlates enable high-fidelity recordings of large and rapid Nav currents due to the combination of gigaohm seal quality recordings and amplifier circuits offering continuous voltage clamp with capacitive and series resistance compensation $(\geq 65 \%)$. All Nav1.x cell lines used in this study have been validated in-house on the QPatch by comparing their voltage dependence, biophysics, and pharmacology to manual and APC data in the literature and information available from APC and cell line vendors and other contract screening companies.

Cells were prepared for QPatch experiments by manual dissociation from T175 cell culture flasks using either 0.05\% trypsin-EDTA (ThermoFisher) or Accutase followed by centrifugation to remove enzyme and debris and resuspension in serum-free media (CHO-III from ThermoFisher or Excel-302 from Sigma-Aldrich). Cells were kept in suspension by constant stirring in the cell hotel on the QPatch before they were washed and resuspended in extracellular recording solution using the Q-fuge unit on-board the QPatch immediately before application to recording wells on the QPlate chip.

Extracellular solution contained (in millimolars) $140 \mathrm{NaCl}$, $5 \mathrm{KCl}, 1 \mathrm{CaCl}_{2}, 1.2 \mathrm{MgCl}_{2}$, and 5 HEPES (pH 7.4), unless inward 
Nav currents were too large, in which case, we reduced the external $\mathrm{Na}^{+}$-permeant cation by making an appropriate admixture from a similar solution in which $140 \mathrm{NaCl}$ was substituted with $140 \mathrm{mM}$ choline chloride. Standard intracellular solution contained (in millimolars) $15 \mathrm{NaCl}, 120 \mathrm{CsF}, 10$ HEPES, 10 EGTA, and 10 HEPES (pH 7.2). The combination of these solutions was also sufficient to isolate inward Nav currents in ND7-23 cells as they effectively inhibited contaminating potassium currents (also present in HEK cells).

The QPatch platform uses static perfusion, where a small volume of recording solution or drug is added to a reservoir on the chip and passes through quartz-lined microfluidic channels to perfuse each cell recording site. This volume is removed by capillary action when the next sample application is made, and excess cells are also removed from the recording chamber by vehicle washes before any compound is added. After achieving the whole-cell configuration, the appropriate vehicle $(0.1 \%$ DMSO v/v or $0.05 \%$ bovine serum albumin [BSA] $\mathrm{w} / \mathrm{v}$ ) was applied to each cell to acquire a control recording of Nav amplitude (usually 4-min period); achieving a stable baseline during the vehicle period is a critical quality control parameter. The potency $\left(\mathrm{IC}_{50}\right.$, the $50 \%$ inhibitory concentration) of compounds against sodium channel subtypes was determined by cumulatively applying three or four escalating concentrations (typically in half log-unit increments) to each cell to construct individual concentration-response relationships. We employed either a single bolus application $(10 \mu \mathrm{L})$ of each test concentration or double addition (at $t=0$ and $t=2 \mathrm{~min}$ ) to expose each cell to a 4-min incubation at each test concentration. Compounds were prepared by dilution (from a $1,000 \times$ stock) in extracellular solution (in appropriate vehicle) to yield a top concentration that was serially diluted into glasslined wells of a microtiter plate compound plate containing extracellular solution and vehicle to avoid any exposure to excess plastic and loss of compound or changes in osmolality. All stock solutions were frozen as aliquots from the master and thawed and used fresh on each day, ensuring only a single freeze-thaw cycle per use.

Currents were elicited from Nav1.3, Nav1.6, and Nav1.7 cell lines and ND7-23 cells using a standard two-pulse voltage protocol. From a holding potential of $-100 \mathrm{mV}$, a $20-\mathrm{ms}$ activating step to the peak of the IV for each Nav1.x channel (0, $-10,-20$, and $-10 \mathrm{mV}$ for Nav1.3, Nav1.6, Nav1.7, and TTX-S, respectively) was applied to assess the effect of compounds on channels opened from the resting (closed) state (Peak 1). The second activating pulse was applied following a 5-s prepulse to a membrane potential that induces $\sim 50 \%$ inactivation of available channels to assess block on the inactivated state of the channel. The half-inactivating potential varied depending on Nav1.x type and tissue culture conditions and was adjusted each day for each target (typically ranging between -70 and $-50 \mathrm{mV}$ ). The two-step voltage protocol was applied at a sweep interval of $0.067 \mathrm{~Hz}$ throughout the duration of the experiment. Leak subtraction was applied between the voltage sweeps, but the length of the voltage protocol only allowed two leak sweeps per epoch, limiting their utility and our use of this method. Rather, sweep subtraction was employed in the QPatch software that enables a single or averaged number of sweeps to be subtracted from all other sweeps in the experiment; applying a saturating dose of $300 \mathrm{nM}$ TTX to each cell at the end of every experiment enabled effective sweep subtraction and removal of capacitive artifacts and contaminating (e.g., outward) currents.

For analysis of hNav1.3, hNav1.6, and hNav1.7 currents in heterologous cells and endogenous TTX-S channels in ND723 cells, the peak inward current amplitude was measured using QPatch assay software (v5.0) by placing cursors over the two test pulses evoking resting- (Peak 1) and half-inactivated state (Peak 2) currents during each voltage protocol sweep. The $\%$ inhibition of peak current was calculated from the mean peak current value for the last three sweeps in each concentration period in the application protocol divided by the average of the last three sweeps recorded at the end of the initial vehicle period. Sigmoidal concentration-response curves (four parameter logistic curve) were fitted to the $\%$ inhibition data using Prism (v6; GraphPad) to determine the mean $\mathrm{IC}_{50}$ value. Fits were constrained to include $0 \%$ and $100 \%$ inhibition at very low and high compound concentrations, respectively, and the hillslope was a free parameter (QC filter was $0.5<\mathrm{nH}>2.0$ ). Data are presented as mean \pm standard deviation (SD) or standard error of the mean (SEM; as indicated) for a minimum of three independent observations. All compounds were tested in at least two independent experiments conducted with separate cell preparations on different days and separate QPlates.

\section{RESULTS}

Confirming Nav1.x Selectivity

of Pharmacological Antagonists

The consensus from previous molecular characterizations of endogenous Nav channels expressed in ND7-23 cells indicates the presence of Nav1.3, Nav1.6, Nav1.7, and possibly Nav1.2 $\alpha$ subunit channel transcripts. ${ }^{32,33}$ To confirm the presence of functional Nav currents carried by these poreforming subunits and determine their relative contribution to total inward Nav current, we would need to make single and additive applications of selective antagonists to individual ND7-23 cells on the QPatch APC platform. As most of these 
ligands had been characterized previously in Xenopus oocytes or by manual patch clamp, we needed to establish their efficacy and Nav1.x selectivity on the QPatch screening platform before using them on ND7-23 cells. Selective ligands were available for Nav1.3, Nav1.6, and Nav1.7 channels, but selective antagonists for Nav1.2 channels were not available at the time of these experiments (e.g., conotoxin TIIIA, tarantula toxins, PaurTx 3 or $C \operatorname{coTx}{ }^{34,35}$ ). To assess the state-dependent inhibition of Nav1.x channels by the chosen ligands, we employed a two-pulse voltage protocol, whereby the first test pulse (Peak 1) was evoked from a negative holding potential (typically $-100 \mathrm{mV}$ ) to assay all channels available from their resting state, and the second test pulse (Peak 2) was delivered $5 \mathrm{~s}$ after a prepulse to a voltage chosen to induce $\sim 50 \%$ inactivation of available Nav1.x channels. There was no return to holding potential after the inactivating prepulse, so this protocol measures ligand potency against both fast and slow inactivated states of each Nav1.x channel. There is a sufficient interval upon return to the negative holding potential after the second pulse and the next application of the two-pulse voltage protocol to allow complete recovery from fast and slow inactivation (data not shown).

Icagen compound 68 (Nav1.3). Icagen, Inc., has published a number of patents and articles describing potent and selective antagonists of Nav1.3 channels. ${ }^{26,36}$ We synthesized several examples and analogs of their arylsulfonamide series as part of our work to discover and design marine-derived inhibitors of ion channels within the EU Framework Program 7 grant-funded MAREX consortium. ${ }^{37}$ Icagen patent compound $68^{26}$ was chosen to be the selective Nav1.3 inhibitor in this study as it is a potent antagonist of Nav1.3 channels with no effects on Nav1.4, Nav1.5, Nav1.6, or Nav1.7 channels. ${ }^{38,39}$ The efficacy of Icagen compound 68 against hNav1.3 channels expressed in CHO cells was determined using the QPatch (Fig. 2 and Table 1). As expected ${ }^{36}$ this compound demonstrates strong state-dependent inhibition of Nav1.3 (Fig. 2B), weakly reducing the amplitude of resting-state (Peak 1) currents with an estimated IC $_{50}$ of $18 \mu \mathrm{M}$ ( $\sim 20 \%$ inhibition at $10 \mu \mathrm{M})$, while dose-dependently inhibiting inactivated (Peak 2) Nav1.3 currents (Fig. 2C) with an $\mathrm{IC}_{50}$ of $56 \mathrm{nM}$ (Fig. 2D). Both resting- and inactivated-state Nav1.3 currents are stable in $0.1 \%$ DMSO vehicle during the course of the QPatch $\mathrm{IC}_{50}$ testing protocol (Fig. 2A), with only a small amount of rundown $(\sim 15 \%)$ seen in inactivated Peak2 currents during the 20-min time course of the experiment, which is typical for whole-cell Nav1.x recordings on QPatch. The average amplitude of inactivated Peak 2 currents at the end of the initial vehicle period in both vehicle (DMSO) and compound 68 datasets ( $\sim 45 \%-50 \%$ of resting-state Peak 1 amplitude, re- spectively) indicates that the prepulse voltage chosen for these Nav1.3 recordings achieved close to the desired level of 50\% steady-state inactivation.

The Nav1.3 selectivity of Icagen compound 68 was previously determined on QPatch (hNav1.5) and in voltage clamp recordings from mammalian Nav1.x channels expressed in Xenopus oocytes (rNav1.4, mNav1.6, hNav1.7) by ourselves and our MAREX collaborators, with no significant effects on resting or inactivated current amplitudes in any of the Nav1.x isoforms tested. ${ }^{39}$

4,9 Anhydro TTX (Nav1.6 vs. Nav1.7). Given the interest in subtype-selective Nav1.x channel ligands for various therapeutic drug discovery efforts and the role of Nav1.6 channels in axonal excitability ${ }^{40}$ and diseases such as multiple sclerosis and epilepsy, ${ }^{11,41}$ it was surprising that an initial finding that the natural TTX metabolite 4,9 anhydro TTX is a selective inhibitor of Nav1.6 channels ${ }^{30}$ had not been confirmed in human Nav1.6 channels. As previous studies had predominantly used rodent Nav1.x channels expressed in Xenopus oocytes or HEK cells, ${ }^{42}$ we first confirmed the potency and selectivity of 4,9 anhydro TTX against human Nav1.x channels expressed in mammalian cells before using it to dissect out endogenous TTX-S channels in ND7-23 cells.

Whole-cell QPatch recordings from HEK cells stably expressing the human Nav1.6 channel revealed inward currents (1-5 nA amplitude) with a rapidly activating and fully inactivating profile evoked by positive test pulses from a negative holding potential of $-100 \mathrm{mV}$. Nav1.6 currents were stable in vehicle control throughout the whole-cell recording (Fig. 3A, $B$ ), while those recorded in cells on the same QPlate, but exposed to 4,9 anhydro TTX, were inhibited in a dose-dependent manner (Fig. 3C). An overlay of macroscopic currents recorded in vehicle and in the presence of increasing concentrations of 4,9 anhydro TTX show no discernible differences in activation or inactivation kinetics (Fig. 3D). The onset of block by 4,9 anhydro TTX (Fig. 3C) was only slightly slower than that seen with TTX, taking several sweeps to reach saturating effect at each concentration. The mean $\mathrm{IC}_{50}$ of $32.9 \mathrm{nM}$ for inhibition of resting hNav1.6 channels (Fig. 3E and Table 1) was slightly less potent than originally obtained for mouse Nav 1.6 channels in oocytes $(7.8 \mathrm{nM}),{ }^{30}$ but fourfold more potent than found by manual patch of rat Nav1.6 channels expressed in HEK cells. ${ }^{42}$ Both the level of maximal block (65\% at $100 \mathrm{nM}$ ) and hillslope (0.6) were slightly lower than in the original oocyte study, but similar to the hillslope for rat Nav1.6 channels in HEK cells. ${ }^{42}$ In determining the $\mathrm{IC}_{50}$, we assumed that maximal block could be achieved at concentrations higher than the $100 \mathrm{nM}$ limit we imposed due to material availability, and the adequate curve fit 


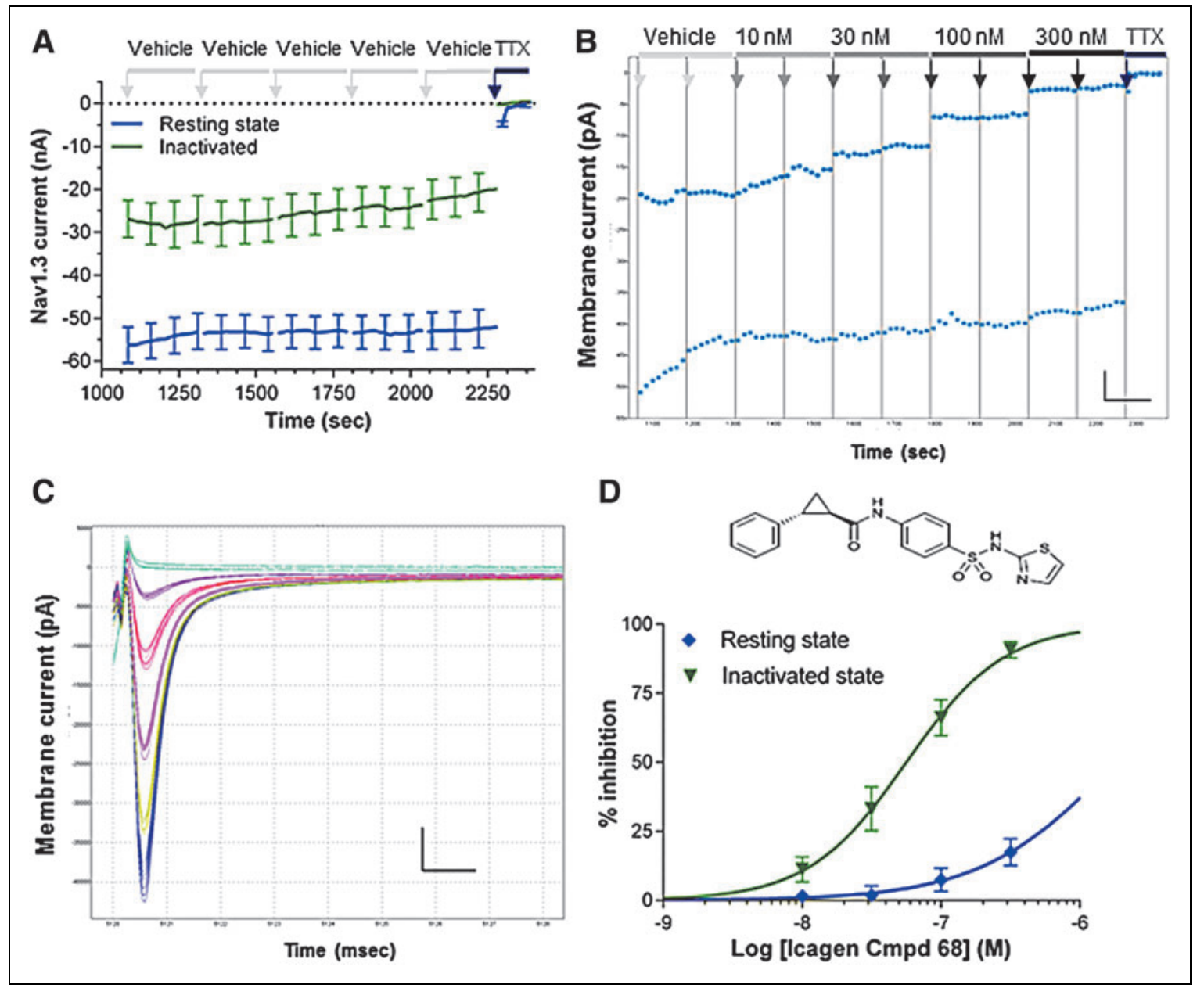

Fig. 2. Characterization of Icagen compound 68 as an Nav1.3 channel inhibitor. The Icagen patent compound (structure in D, inset) was applied to CHO cells stably expressing the hNav1.3 channel to determine its potency and state dependency. (A) Average macroscopic Nav1.3 currents evoked in $0.1 \%$ DMSO vehicle (multihole X-plates) from resting state (Peak 1 , blue line) and following a prepulse to $\sim 50 \%$ inactivation membrane potential (Peak 2, green line) from $N=6$ cells ( \pm SEM for every sixth sweep) show acceptable current stability (Peak 2 rundown $<1 \%$ / min) during the $\sim 20$-min duration of the whole-cell experiment. Peak 2 shows $\sim 50 \%$ inactivation (amplitude) compared with Peak 1 . Each dose application epoch of the $\mathrm{IC}_{50}$ experiment is separated by a gap due to pipette scheduling across different wells on the QPlate and ends with application of $300 \mathrm{nM}$ TTX to block remaining current. Arrows show 'liquid addition' timing of each compound application. (B) Repeat of the experiment in (A), but with increasing concentrations of Icagen compound 68 applied to a single cell (indicated by arrows) after the initial vehicle stabilization period, showing the dose- and state-dependent inhibition of inactivated Peak 2 current $(\bullet)$ compared with minimal effects on resting Peak 1 current $(\boldsymbol{\square})$. In this and all other QPatch raw data current-time (IT) plots, current amplitude (in picoampere or in this case nanoampere) is plotted on the $y$-axis and experimental time (in s) on the $\mathrm{x}$-axis. Scale bar shows $5 \mathrm{nA}$ on $\mathrm{y}$-axis and $100 \mathrm{~s}$ on $\mathrm{x}$-axis. (C) Example of inactivated-state Peak 2 currents (following a 5 -s prepulse to $\sim 50 \%$ inactivating membrane potential) in a typical cell showing dose-dependent inhibition of inward currents (vehicle = blue traces, $10 \mathrm{nM}=$ green, $30 \mathrm{nM}=$ pink, $100 \mathrm{nM}=$ red, and $300 \mathrm{nM}=$ purple); Cyan traces are in the presence of $300 \mathrm{nM}$ TTX, showing complete block of all fast inward currents. In this and all other raw current data traces exported from QPatch software, current amplitude (in picoampere) is plotted on the y-axis and time (ms) on the $\mathrm{x}$-axis. Scale bar shows $5 \mathrm{nA}$ on the $y$-axis and $1 \mathrm{~ms}$ on the $x$-axis. (D) Plot of mean ( $N=4$ cells, $\pm S E M) \%$ inhibition data allows fitting of $I C_{50}$ curves (sigmoidal dose-response) for Icagen compound block of resting-state Nav1.3 currents ( Peak 1, estimated $18 \mu \mathrm{M}$ ) and inactivated state ( $\nabla$ Peak 2, $56 \mathrm{nM}$ with hillslope of 1.2). Inset: structure of Icagen compound 68 . CHO, Chinese hamster ovary; DMSO, dimethyl sulfoxide; $I_{50}, 50 \%$ inhibitory concentration; SEM, standard error of the mean; TTX, tetrodotoxin.

to the mean data in Figure $3 E\left(r^{2}=0.94\right.$, data not shown $)$ indicates that this is a reasonable assumption when using a somewhat shallow hillslope.

We observed that the potency of 4,9 anhydro TTX was significantly higher for Nav1.6 currents evoked after an in- activating prepulse (Peak $2 \mathrm{IC}_{50}$ of $4.3 \mathrm{nM}$, Fig. $3 E$ ) than that for block of the open state evoked from a negative resting potential. This is indicative of a state-dependent mechanism of 4,9 anhydro TTX to bind with higher affinity to a conformational state associated with the inactivated state, 
Table 1. Summary of Nav1.x-Selective Pharmacology and ND7-23 Cell TXX-S Current Components

$$
\text { Nav1.X } I C_{50}(n M)
$$

ND7-23 TIX-S

\begin{tabular}{l|l|l|l|l|l} 
hNav1.3 & hNav1.6 & hNav1.7 & $I_{50}$ (nM) & \% Inhibition \\
\hline
\end{tabular}

Icagen compound 68

\begin{tabular}{r|r|r|c|c|c}
\hline Peak 1 & 18,000 & $\mathrm{NE}^{\mathrm{b}}$ & $\mathrm{NE}^{\mathrm{c}}$ & $\mathrm{NE}$ & 0.8 \\
\hline Peak 2 & 56 & $\mathrm{NE}^{\mathrm{b}}$ & $\mathrm{NE}^{\mathrm{c}}$ & $>10,000$ & -0.5 \\
\hline
\end{tabular}

4,9 Anhydro TTX

\begin{tabular}{c|c|c|c|c|c}
\hline Peak 1 & ND & 32.9 & 1,600 & $>300$ & 15.2 \\
\hline Peak 2 & ND & 4.3 & $>300$ & $>100$ & 33.7 \\
\hline
\end{tabular}

Protoxin-II

\begin{tabular}{r|c|c|c|c|c}
\hline Peak 1 & ND & ND & 3.7 & 7.2 & 61.7 \\
\hline Peak 2 & ND & ND & 2.3 & 5.7 & 63.6 \\
\hline
\end{tabular}

aPercent of inhibition produced by selective concentrations of Icagen compound 68 (300 nM), 4,9 anhydro TX (100 nM), or Protoxin-II (10 nM) applied to different cells.

${ }^{b}$ Determined against mouse Nav1.6 channels. $^{39}$

'From Ref. ${ }^{39}$

$I_{50}, 50 \%$ inhibitory concentration; ND, not determined; NE, no effect; $T X-S$, tetrodotoxin-sensitive.

similar to observations with TTX. ${ }^{43,44}$ Thus, to simplify the use of 4,9 anhydro TTX as a ligand to discriminate between Nav1.x channel subtypes in ND7-23 cells, we concentrated on its effects on the resting state of Nav1.x channels.

As molecular profiling indicates that Nav1.7 is the only other major TTX-S Nav subtype expressed in ND7-23 cells, we limited our selectivity profiling of 4,9 anhydro TTX to human Nav1.6 and Nav1.7 channels. Inward currents evoked in HEK cells stably expressing hNav1.7 channels using the same voltage protocol described above exhibit rapid activation and inactivation kinetics (Fig. 4C, E). Both hNav1.7 current amplitude (Fig. 4A) and kinetics are stable in vehicle, indicating that the rapid inward currents of $\leq 5 \mathrm{nA}$ amplitude are well clamped in single-hole gigaseal recordings on the QPatch platform. All inward currents in hNav1.7-expressing cells were completely inhibited by addition of $300 \mathrm{nM}$ TTX to each cell at the end of the experiment (Fig. 4A-E). Repeating the experiment of Figure $3 C$ on cells expressing hNav1.7 channels shows no inhibition of resting-state Nav1.7 current amplitude by 4,9 anhydro TTX up to a test concentration of $100 \mathrm{nM}$ (Fig. 4B, C), whereas the same concentration reduced hNav1.6 currents by $>50 \%$. Increasing the 4,9 anhydro dose range by 30 -fold reveals dose-dependent inhibition of hNav1.7 currents (Fig. 4D, E) to yield an $\mathrm{IC}_{50}$ for resting-state Peak 1 currents of $1.62 \mu \mathrm{M}$
(Fig. 4F). As with hNav1.6 channels, the kinetics of hNav1.7 channel block by 4,9 anhydro TTX are slightly slower than seen with TTX (300 nM) added at the end of each experiment.

These data reveal that 4,9 anhydro TTX is a potent inhibitor of human Nav1.6 channels with 50-fold selectivity over hNav1.7 channels (Table 1), confirming the selectivity shown previously in rodent Nav1.x channels expressed in oocytes. ${ }^{30}$ We conclude that we can use 4,9 anhydro TTX at a moderate concentration of $100 \mathrm{nM}$ to distinguish between the activity of Nav1.6 and Nav1.7 channels in ND7-23 cells.

Protoxin-II (Nav1.7). The Nav1.7 selectivity of Protoxin-II has been established by several groups using manual patch clamp of human Nav1.x channels expressed in mammalian cells, ${ }^{27}$ so we first confirmed its efficacy against human Nav1.7 channels in HEK cells on the QPatch before using it as a tool to probe Nav currents in ND7-23 cells. Protoxin-II is not as water soluble as TTX, but to prevent any nonspecific loss of peptide activity or absorption onto glass surfaces, we added 0.05\% BSA to all solutions used in the QPatch experiments with this ligand. Accordingly, we first determined that 0.05\% BSA vehicle would not affect Nav current stability or create artifacts (e.g., bubbles) with the small volumes and laminar flow channels employed on the QPlates. Figure 5A and $B$ illustrates that hNav1.7 currents in HEK cells (and TTX-S currents in ND7-23 cells, Fig. 8A, B) are stable in the presence of 0.05\% BSA with no change in inward current amplitude or kinetics during the course of a 20-min QPatch experiment, thereby enabling reliable screening and pharmacological profiling of agents dissolved in this vehicle. Rapidly activating and inactivating resting-state hNav1.7 currents (1.5-6 nA peak amplitude) were well clamped by the QPatch (Fig. 5A, C). Nav1.7 currents exhibited dose-dependent inhibition by nanomolar concentrations of Protoxin-II (Fig. 5C, D), yielding an $\mathrm{IC}_{50}$ of $3.7 \mathrm{nM}$ for Peak 1 currents (Fig. 5E). Inactivatedstate Peak 2 Nav1.7 currents were inhibited by Protoxin-II (Fig. 5D) with little state dependence, yielding an average $\mathrm{IC}_{50}$ of $2.3 \mathrm{nM}$ (Fig. 5E, Table 1).

To maximize current block by this slow-acting toxin, we employed triple additions of each test concentration. The rate of block of Nav1.7 currents by Protoxin-II increased with higher peptide concentrations and saturated at $>90 \%$ inhibition at the top concentration tested of $10 \mathrm{nM}$. Using higher concentrations of Protoxin-II to achieve full block of Nav1.7 was avoided for two reasons. First, higher doses $(\geq 100 \mathrm{nM})$ can induce sustained currents through Nav1.7 channels. ${ }^{45}$ Second, although the 70- to 100-fold selectivity of Protoxin-II for Nav1.7 over other TTX-S channels is exceptional, ${ }^{46}$ Nav1.6 is the second most sensitive Nav1.x channel to Protoxin-II with 

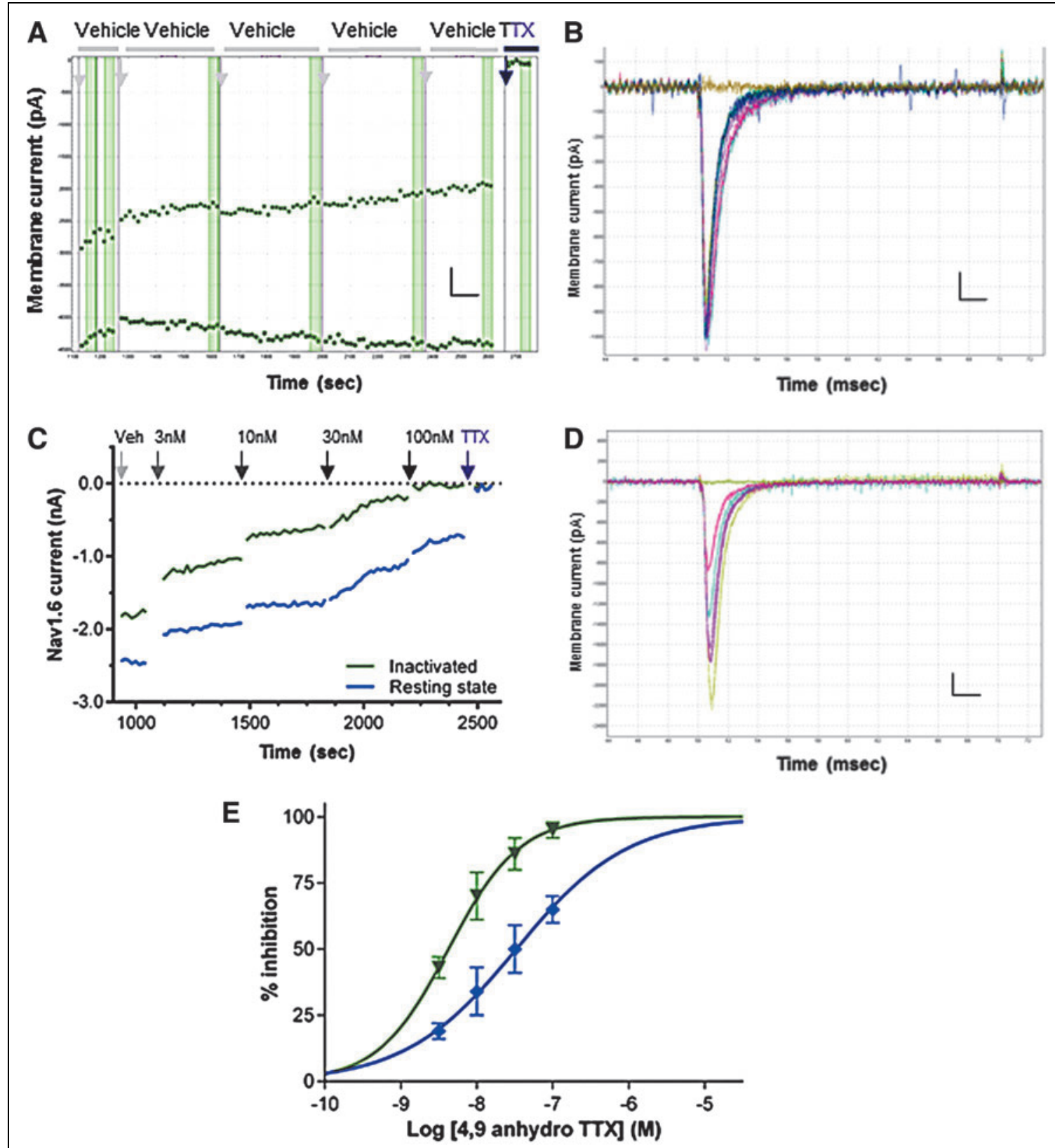

Fig. 3. Potency of 4,9 anhydro TTX against human Nav1.6 channels. (A, B) Heterologous hNav1.6 currents (single-hole QPlates) are stable throughout a 20 -min experiment in $0.1 \%$ DMSO vehicle when evoked from resting state (B) or $\sim 50 \%$ inactivating membrane potentials (Peak 2, in A). Peak 2 currents were $40 \%$ inactivated at the end of the vehicle period in $0.1 \%$ DMSO control cells and $53 \%$ of the amplitude of Peak 1 currents during initial vehicle period in cells exposed to 4,9 anhydro TTX. Scale bar in (A) shows 500 pA on the $y$-axis and $100 \mathrm{~s}$ on the $\mathrm{x}$-axis. TTX (300 nM) added at the end of every experiment completely inhibits inward currents (A, olive traces in B). Scale bar in (B) shows $100 \mathrm{pA}$ on the $\mathrm{y}$-axis and $2 \mathrm{~ms}$ on the x-axis. Arrows in A (and C) show timing of addition of vehicle or compound. (C, D) After a brief stabilization period in vehicle, addition of increasing concentrations of 4,9 anhydro TTX dose-dependently inhibits hNav1.6 currents from resting or inactivated potentials (blue and green traces in C, respectively) without altering their kinetics (resting-state current, D). Effects of $3 \mathrm{nM}$ (purple traces), $10 \mathrm{nM}$ (pink), $30 \mathrm{nM}$ (cyan), and $100 \mathrm{nM} 4,9$ anhydro TTX (red) on resting current in a typical cell are shown in (D) as well as control currents in vehicle (olive traces) and after application of $300 \mathrm{nM}$ TTX (green); as $300 \mathrm{nM}$ TTX completely inhibits Nav1.6 currents (A-C), we subtract these sweeps offline to remove capacitance artifacts remaining after $\mathrm{Cm}$ and Rs compensation. Scale bar in (D) shows $200 \mathrm{pA}$ on the $\mathrm{y}$-axis and $2 \mathrm{~ms}$ on the $\mathrm{x}$-axis. (E) The $\mathrm{IC}_{50}$ of 4,9 anhydro TTX block of resting-state (32.9 $\mathrm{nM}$, $\left.\diamond\right)$ and inactivated-state hNav1.6 channels $(4.3 \mathrm{nM}, \boldsymbol{\nabla})$ was obtained by fitting a sigmoidal dose-response curve to the mean $\pm \mathrm{SEM}(N=4$ cells) $\%$ inhibition data (variable hillslope parameters of 0.59 and 0.94 , respectively). 
an $\mathrm{IC}_{50}$ of $26 \mathrm{nM} .^{27}$ This profile creates limited selectivity of Protoxin-II for Nav1.7 over other TTX-S channels known to be expressed in ND7-23 cells and puts an upper limit of $10 \mathrm{nM}$ on the concentration of Protoxin-II that can be used to maintain specificity for Nav1.7 channels.

The fact that our QPatch validation studies were conducted on human Nav1.7 channels, but the profiling of endogenous Nav1.x channels was to be carried out in a rodent cell line, raises the potential for species differences in Nav1.x selectivity. However, previous data ${ }^{27,45}$ indicate that there is no significant difference in the potency or Nav1.x selectivity of Protoxin-II between human and rat TTX-S sodium channels. In addition, the potency for 4,9 anhydro TTX inhibition of hNav1.6 channels was similar in this study to that obtained for mouse Nav1.6 channels ${ }^{30}$ and although the potency against rat Nav1.6 channels expressed in HEK cells was lower
(122 nM), those authors selected $100 \mathrm{nM} \mathrm{4,9}$ anhydro TTX as the specific concentration. ${ }^{42}$ Taking these observations together, a concentration of $100 \mathrm{nM} \mathrm{4,9}$ anhydro TTX and $10 \mathrm{nM}$ Protoxin-II was considered to be selective for Nav1.6 and Nav1.7 channels, respectively, during subsequent experiments to determine the profile of functional Nav1.x channels in ND7-23 cells.

\section{Determining the Functional Repertoire} of Nav1.x Channels in ND7-23 Cells

Undifferentiated ND7-23 cells can be dissociated into single-cell suspensions that resemble HEK cell preparations and perform extremely well on APC platforms, yielding a high proportion of gigaohm seals that successfully convert into stable whole-cell recordings with good access and low series resistance suitable for high-fidelity recordings of endogenous

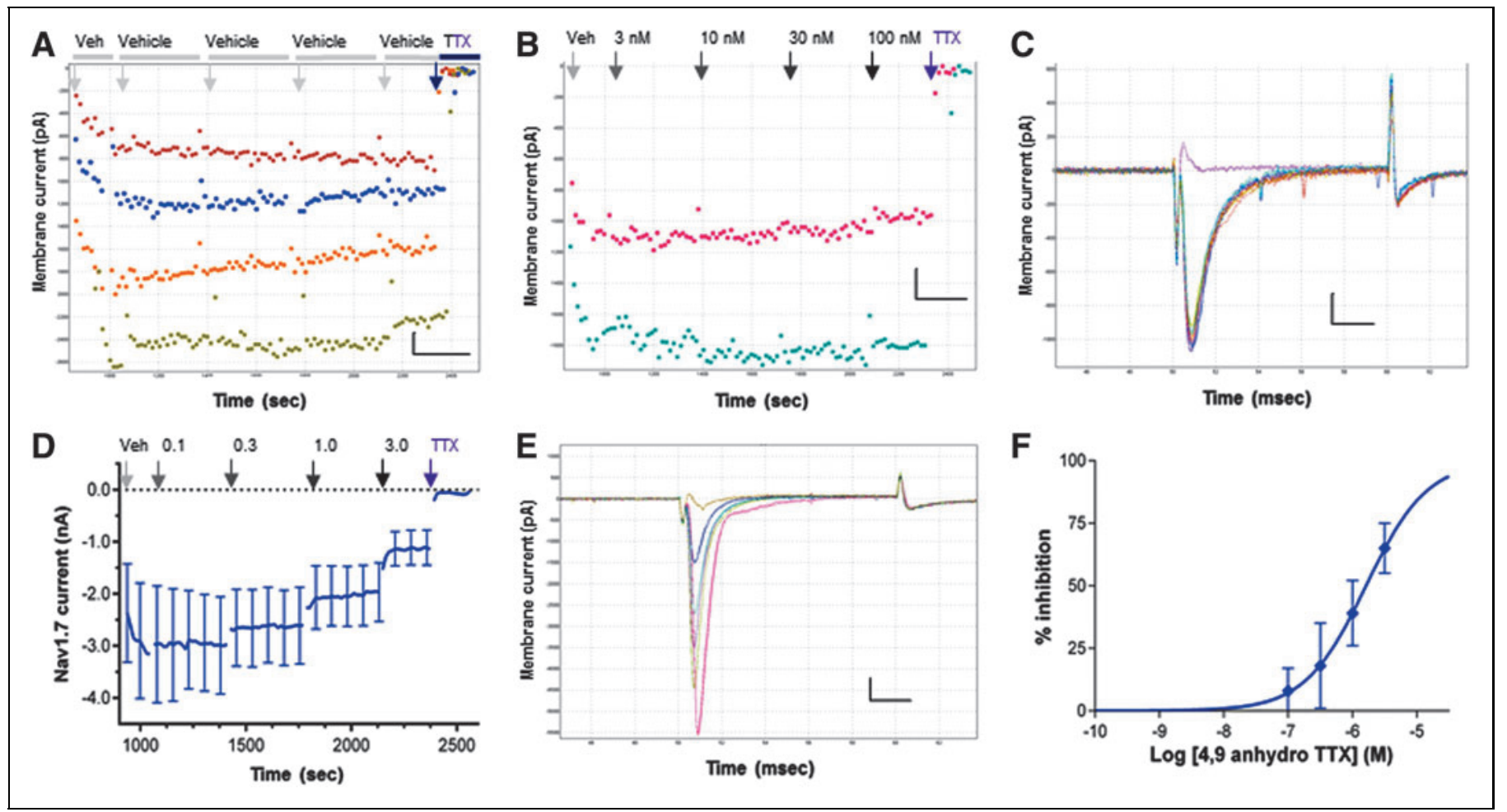

Fig. 4. Potency and selectivity of 4,9 anhydro TTX against human Nav1.7 channels. (A) Resting-state currents of hNav1.7 channels expressed heterologously in HEK cells are stable in $0.1 \%$ DMSO vehicle on the QPatch and completely blocked by $300 \mathrm{nM}$ TTX. Each set of colored symbols represents a different cell. Scale bar shows 200 pA on the y-axis and $200 \mathrm{~s}$ on the x-axis. Arrows (also in B and D) indicate timing of vehicle or compound additions. (B, C) Exposure of hNav1.7-expressing cells to concentrations of 4,9 anhydro TTX that inhibit hNav1.6 channels has no effect on resting-state Peak 1 current amplitude (B) or kinetics (C), but all inward currents are completely blocked by addition of $300 \mathrm{nM} \mathrm{TTX}$ at the end of the experiment (purple traces in C). (B) Shows the time course of Nav1.7 current amplitude in two cells exposed to 3-100 nM 4,9 anhydro TTX, followed by $300 \mathrm{nM}$ TTX. Scale bar in (B) shows 200 pA on the $y$-axis and $200 \mathrm{~s}$ on the x-axis, and $200 \mathrm{pA}$ and $2 \mathrm{~ms}$ in (C). (D, E) Application of 30-fold higher concentrations of 4,9 anhydro TTX produces dose-dependent inhibition of resting-state Peak 1 currents. Mean resting-state Nav1.7 current amplitude ( \pm SEM from $N=3$ cells) plotted over time is shown in (D), and raw data from a single cell in (E; red traces = vehicle, green $=0.1 \mu \mathrm{M}$, purple $=0.3 \mu \mathrm{M}$, cyan $=1 \mu \mathrm{M}$, blue $=3 \mu \mathrm{M}$, and brown $=300 \mathrm{nM} \mathrm{TTX}$ ). Three hundred nanomolars of TTX applied to each cell at the end of the experiment completely blocked all inward currents. Scale bar in (E) shows $500 \mathrm{pA}$ on the $\mathrm{y}$-axis and $2 \mathrm{~ms}$ on the $\mathrm{x}$-axis (F) The dose-response curve of hNav1.7 channel inhibition by 4,9 anhydro TTX reveals an $\mathrm{IC}_{50}$ of $1.62 \mu \mathrm{M}$ (mean $\pm \mathrm{SEM}$ from $N=3$ cells). HEK, human embryonic kidney. 
ROGERS ET AL.

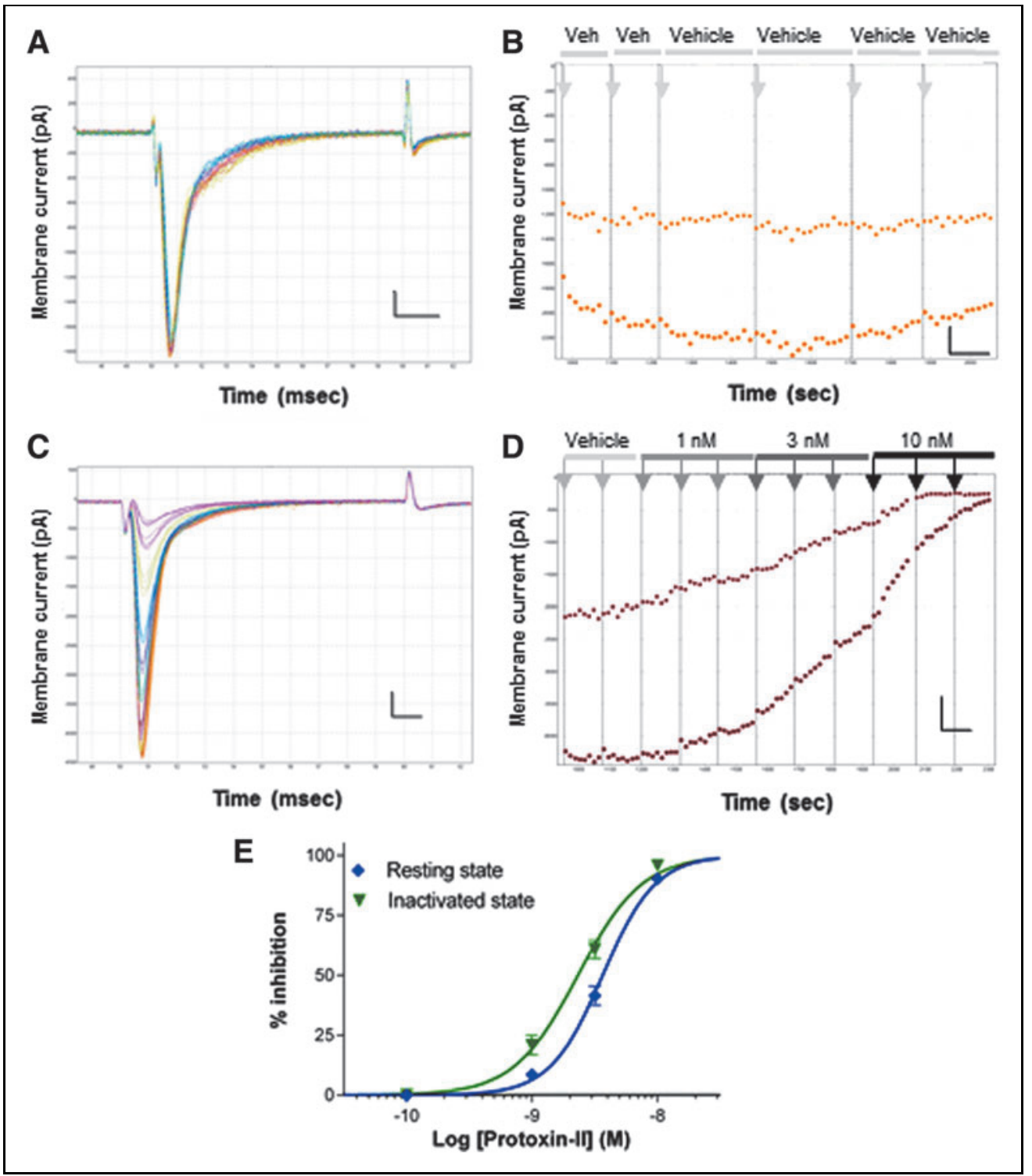

Fig. 5. Confirming potency and state dependency of Protoxin-II for hNav1.7 channels on QPatch. (A, B) Resting- (A) and inactivated-state currents through hNav1.7 channels expressed in HEK cells are stable in $0.05 \%$ BSA vehicle. Resting-state current (• in B) exhibits slight runup during the vehicle stabilization period, while inactivated-state Peak 2 current $(\boldsymbol{B}$ in $\mathbf{B})$ is $\sim 50 \%$ of Peak 1 amplitude and stable throughout the 20 -min experiment. Scale bar in (A) is $200 \mathrm{pA}$ on the $\mathrm{y}$-axis and $2 \mathrm{~ms}$ on the $\mathrm{x}$-axis and $200 \mathrm{pA}$ on the $\mathrm{y}$-axis and $100 \mathrm{~s}$ on the $\mathrm{x}$-axis in (B). Arrows (also in D) indicate timing of vehicle or compound additions. (C) Application of nanomolar concentrations of Protoxin-II (in 0.05\% BSA to prevent nonspecific loss of activity) dose-dependently inhibits resting-state Peak 1 current in an exemplar cell; Protoxin-II was applied $3 \times$ at each test concentration (vehicle =orange traces; $1 \mathrm{nM}=$ olive, purple, and brown traces; $3 \mathrm{nM}=$ cyan, blue, and light blue traces; $10 \mathrm{nM}=$ green, purple, and pink traces). Scale bar is $500 \mathrm{pA}$ on the $y$-axis and $1 \mathrm{~ms}$ on the $x$-axis. (D) IT plot from the cell shown in (C) illustrates current stability during the vehicle period and slow dose-dependent block of resting-state Peak $1(\bullet)$ and inactivated-state Peak 2 currents $(\boldsymbol{\square})$ at low nanomolar concentrations, with near maximal efficacy at the highest dose of $10 \mathrm{nM}$. Scale bar is $500 \mathrm{pA}$ on the $\mathrm{y}$-axis and $100 \mathrm{~s}$ on the $\mathrm{x}$-axis. (E) Plot of average \% inhibition (mean $\pm \mathrm{SEM}$ from $N=3$ cells) of resting-state $(\diamond)$ and inactivated-state $(\nabla)$ currents against Protoxin-II concentration reveals a Peak $1 \mathrm{IC}_{50}$ of $3.65 \mathrm{nM}$ and a Peak $2 \mathrm{IC} \mathrm{C}_{50}$ of $2.32 \mathrm{nM}$. Sigmoidal least squares fit with hillslopes of 2.1 and 1.7 for resting- and inactivated-state curves, respectively. BSA, bovine serum albumin.

ion channel currents. In contrast to QPatch recordings from CHO and HEK cells expressing heterologous Nav channels that can show runup or rundown of resting and inactivated current amplitude (e.g., Figs. 2A, 4A, and 5C), endogenous Nav currents in dissociated ND7-23 cells are remarkably stable throughout the 20-min duration of a typical $\mathrm{IC}_{50}$ experiment (Figs. 6A, 7A, and 8C). A more negative resting membrane potential in neuronal ND7-23 cells could account for this stability as it allows a greater proportion of endogenous Nav channels to reside in a fully available resting state with little 

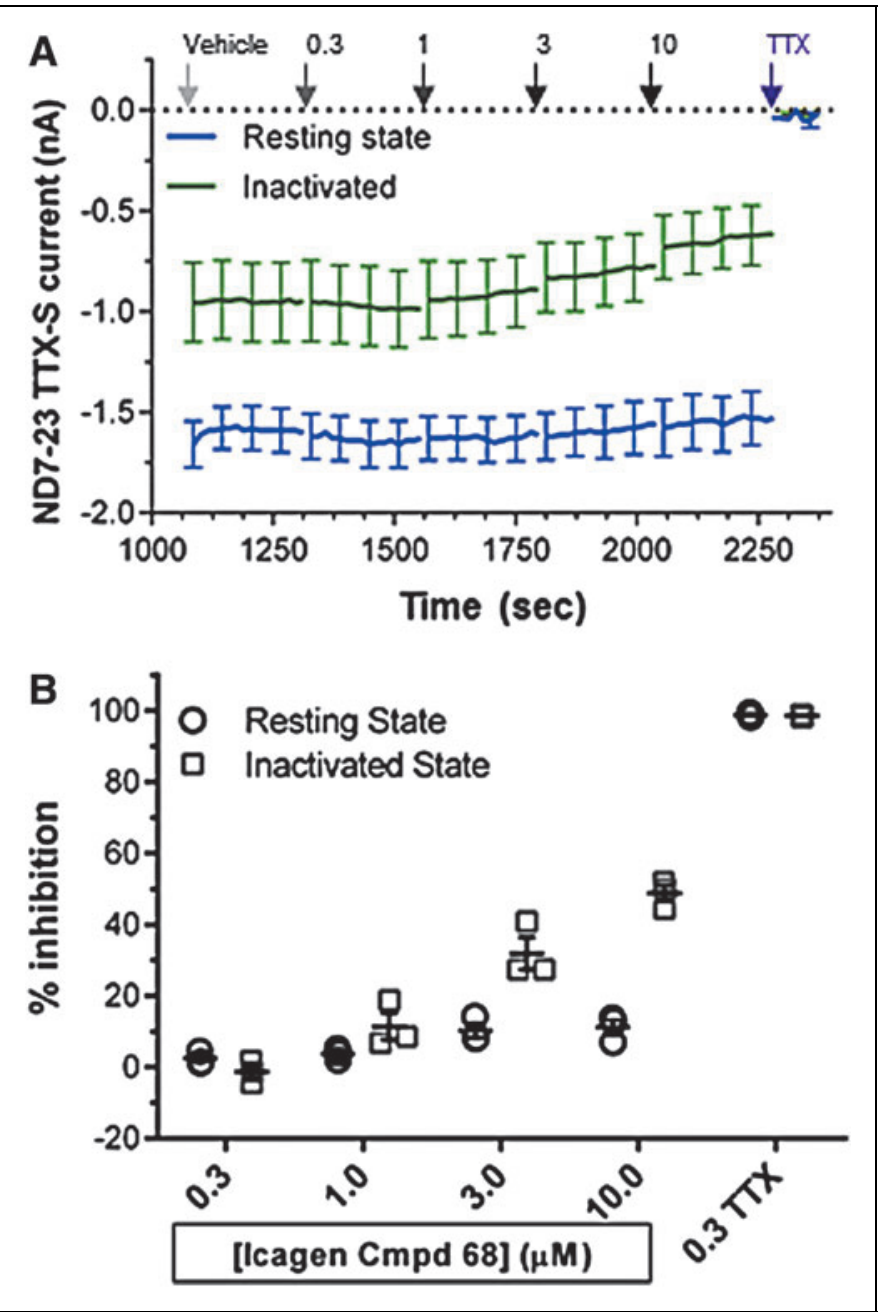

Fig. 6. Effect of an Nav1.3 inhibitor on TTX-S currents in ND7-23 cells. (A) Current-time plot of average (mean $\pm \mathrm{SEM}$ from $N=5$ cells) resting-state Peak 1 (blue) and inactivated-state Peak 2 (green) TTX$\mathrm{S}$ current amplitude recorded in ND7-23 cells in vehicle $(0.1 \%$ DMSO) and increasing concentrations of Icagen compound 68 (application times and concentrations in $\mu \mathrm{M}$ indicated by arrows), illustrating state-dependent preference for inhibition of inactivatedstate currents. Note that Peak 2 currents were $\sim 50 \%$ inactivated in the vehicle stabilization period. All currents remaining after incubation in $10 \mu \mathrm{M}$ of Icagen compound are blocked by $300 \mathrm{nM}$ TTX. (B) Histogram illustrating mean \% inhibition values for resting-state (black open circles) and inactivated-state currents (gray open squares) after exposure to indicated concentrations of Icagen compound 68 , followed by $300 \mathrm{nM}$ TTX; values for individual cells are shown by each symbol, together with mean \pm SEM error bars $(N=3$ cells). Inhibition of TTX-S currents was always $<50 \%$, so data could not be fitted with a reliable $\mathrm{IC}_{50}$ curve. TTX-S, tetrodotoxin-sensitive.

inactivation. In contrast, the depolarized resting potential in CHO and HEK cells leads to accumulation of heterologous Nav channels in a long-lived inactivated state from which they slowly recover at the beginning of a whole-cell recording (runup) and quickly return to during the experiment (rundown).
Consistent with previous molecular, biophysical, and pharmacological studies, our results show that all of the endogenous Nav currents in ND7-23 cells are carried by TTX-S channels. Application of $300 \mathrm{nM}$ TTX at the end of each experiment in every ND7-23 cell inhibited 98.4\% $\pm 2.0 \%$ (mean \pm SD from $N=33$ cells) of peak inward resting-state Peak 1 currents and $300 \mathrm{nM}$ TTX inhibited inactivated-state Peak 2 currents to a similar extent (Figs. 9 and 10B).

Application of a high, but moderately selective, concentration $(300 \mathrm{nM})$ of the Icagen Nav1.3 compound 68 produced little discernible effect on either resting-state or inactivated-state TTX-S currents in ND7-23 cells (Fig. 6), even though this was 5X higher than the inactivated-state $\mathrm{IC}_{50}$ determined with a similar voltage protocol using heterologous Nav1.3 channels (Fig. 2). The full four-point cumulative $\mathrm{IC}_{50}$ application protocol exposed ND7-23 cells to half log-unit increases in concentration of the Icagen compound, and as these reached levels that were no longer selective for Nav1.3 channels, there was an incremental increase in inward current inhibition, with the expected statedependent profile favoring inhibition of inactivated-state (Peak 2) currents compared with resting-state (Peak 1) currents (Fig. 6B). As the maximum $\%$ inhibition values were $<50 \%$ and the higher concentrations were no longer selective for Nav1.3 channels (especially inactivated Peak 2 data), we did not attempt to measure potency or calculate an $\mathrm{IC}_{50}$ from this dataset.

We then determined the fraction of endogenous TTX-S currents carried by Nav1.6 channels by applying selective concentrations of 4,9 anhydro TTX $(10-100 \mathrm{nM})$. As noted above, inward currents evoked in ND7-23 cells from resting (Fig. 7A, C) and half-inactivated membrane potentials (Fig. 7B) were remarkably stable during control experiments in the presence of vehicle ( $0.1 \%$ DMSO) and during the initial control period of the $\mathrm{IC}_{50}$ application protocol before application of 4,9 anhydro TTX. There were minor effects of 10 and $30 \mathrm{nM} 4,9$ anhydro TTX on resting-state Peak 1 and inactivated-state Peak 2 currents (Fig. 7B), while the top concentration tested of $100 \mathrm{nM} 4,9$ anhydro TTX (which is $\sim 3 \times$ the Nav1.6 IC $_{50}$, but remains selective for Nav1.6 over Nav1.7 channels) produced significant inhibition of both resting-state and half-inactivated state currents. Although there is clear evidence for dose-dependent inhibition of ND7-23 cell TTX-S currents by selective concentrations of 4,9 anhydro TTX, the low \% inhibition values precluded us from determining accurate potency data or calculating an $\mathrm{IC}_{50}$ value. However, the moderate effects of 4,9 anhydro TTX were reasonably reversible after application of small volumes of vehicle to the QPlate recording chamber (Fig. 7B), suggesting they were not due to rundown or other artifacts.

Protoxin-II is less water soluble than TTX and to preserve its low nanomolar potency in the small volume static flow 


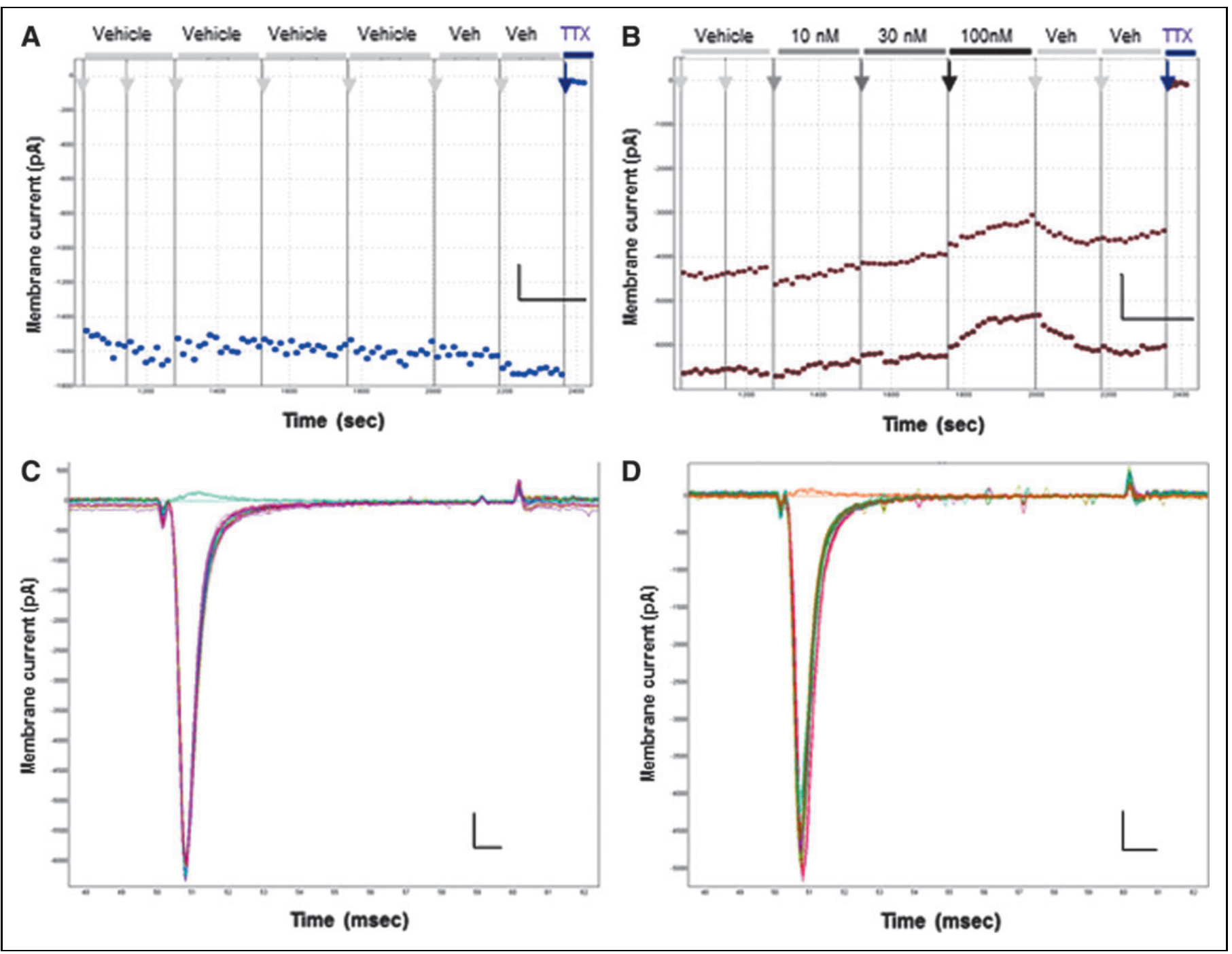

Fig. 7. Inhibition of TTX-S currents in ND7-23 cells by selective Nav1.6 antagonist 4,9 anhydro TTX. (A) Current-time plot of resting-state Peak 1 TTX-S current recorded in a typical ND7-23 cell in vehicle (0.1\% DMSO applications shown by gray arrows) showing stable current amplitude and lack of any initial runup or rundown in these cells. All inward currents are blocked by application of $300 \mathrm{nM}$ TTX at the end of the experiment (purple arrow). Scale bar is $200 \mathrm{pA}$ on the $\mathrm{y}$-axis and $200 \mathrm{~s}$ on the $\mathrm{x}$-axis. (B) In another cell, the application (indicated by arrows) of increasing concentrations of 4,9 anhydro TTX shown to significantly inhibit Nav1.6 channels has little effect on resting-state Peak 1 ( $\bullet$ ) or inactivated-state Peak 2 currents ( $\square$ ), producing a maximal inhibition of $\sim 20 \%$ at $100 \mathrm{nM}$ before washout in vehicle, and then complete block by $300 \mathrm{nM} \mathrm{TTX}$ at the end of the experiment. Scale bar is $1 \mathrm{nA}$ on the $\mathrm{y}$-axis and $200 \mathrm{~s}$ on the x-axis. Inhibition of TTX-S currents by 4,9 anhydro TTX was always $<50 \%$, so data could not be fitted with a reliable $\mathrm{IC}_{50}$ curve. (C) Exemplar resting-state Peak 1 TTX-S current traces in $0.1 \%$ DMSO vehicle exhibit stable amplitude and kinetics during an $\sim 20$-min whole-cell experiment. As all inward currents are completely blocked by $300 \mathrm{nM}$ TTX (cyan traces), the average of the last three sweeps in TTX has been subtracted from all other sweeps to remove capacitance transients. Scale bar is 500 pA on the $y$-axis and 1 ms on the x-axis. (D) Overlay of resting-state Peak 1 currents in another cell in vehicle (red traces) and after exposure to $10 \mathrm{nM}$ (green traces), $30 \mathrm{nM}$ (purple traces), and $100 \mathrm{nM}$ 4,9 anhydro TTX (blue traces), followed by washoff in vehicle (olive traces), and then complete inhibition by $300 \mathrm{nM}$ TTX (orange traces). Sweeps recorded in TTX have been subtracted to remove capacitance transients remaining after online $\mathrm{Cm}$ and Rs compensation. Scale bar is $500 \mathrm{pA}$ on the $\mathrm{y}$-axis and $1 \mathrm{~ms}$ on the $\mathrm{x}$-axis.

chambers on the QPlate, we used 0.05\% BSA as the vehicle in all external solutions; all activity of Protoxin-II was lost in the absence of BSA as a protein carrier. It was therefore necessary to first establish that the BSA vehicle had no effect on endogenous TTX-S currents in ND7-23 cells on the QPatch platform. Figure $8 A$ and $C$ shows that both resting-state Peak 1 and half-inactivated Peak 2 currents remain stable throughout the 20-min experimental recording period in the presence of $0.05 \%$ BSA, as seen previously for Nav current recordings from ND7-23 cells made in external saline and 


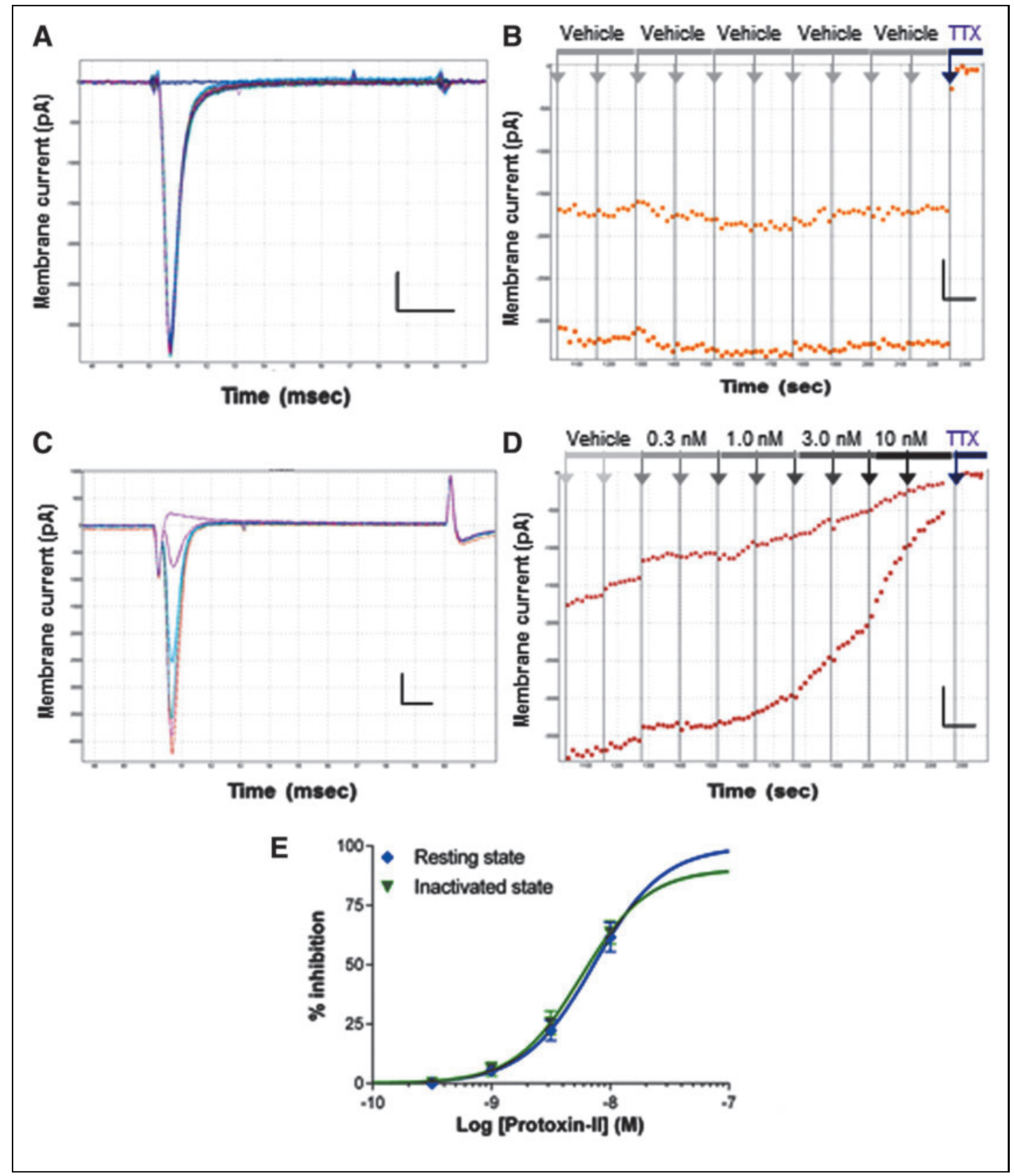

Fig. 8. Inhibition of TTX-S currents in ND7-23 cells by selective Nav1.7 antagonist Protoxin-II. (A, B) TTX-S currents in ND7-23 cells are stable in $0.05 \%$ BSA vehicle. Resting-state (raw current traces in A and $\boldsymbol{\bullet}$ in $\mathbf{B}$ ) and inactivated-state currents ( $\boldsymbol{\square}$ in $\mathbf{B}$ ) are stable throughout the 20-min whole-cell recording, and inactivated-state Peak 2 current is $\sim 50 \%$ of Peak 1 amplitude; current is blocked by $300 \mathrm{nM}$ TTX applied at the end of the experiment (blue traces in A, purple arrow in B). Scale bar in (A) is $500 \mathrm{pA}$ on the $\mathrm{y}$-axis and $2 \mathrm{~ms}$ on the $\mathrm{x}$-axis, and $500 \mathrm{pA}$ and $100 \mathrm{~s}$ in (B). (C) Application of nanomolar concentrations of Protoxin-II (in $0.05 \%$ BSA to preserve peptide activity and prevent absorption to plastic or glass surfaces) dose-dependently inhibits resting-state Peak 1 current in an exemplar cell; vehicle = brown traces, $0.3 \mathrm{nM}=$ purple, $1 \mathrm{nM}=$ cyan, $3 \mathrm{nM}=$ blue, $10 \mathrm{nM}=$ purple; $300 \mathrm{nM} \mathrm{TTX}=$ pink traces). Scale bar is $500 \mathrm{pA}$ on the $\mathrm{y}$ axis and $1 \mathrm{~ms}$ on the x-axis. (D) Time course plot from another cell illustrates current stability in vehicle and slow dose-dependent block of resting-state $(\bullet)$ and inactivated-state currents $(\boldsymbol{\square})$ at low nanomolar concentrations (concentrations and applications indicated by arrows) and almost complete block after a double addition of $10 \mathrm{nM}$ Protoxin-II before application of a saturating dose of $300 \mathrm{nM}$ TTX. Scale bar is $500 \mathrm{pA}$ on the $\mathrm{y}$-axis and $100 \mathrm{~s}$ on the $\mathrm{x}$-axis. (E) Plot of average \% inhibition (mean $\pm \mathrm{SEM}$ from $N=6$ cells) of resting-state ( $\bullet$ ) and inactivated-state $(\boldsymbol{\nabla})$ current against Protoxin-II concentration reveals a Peak $1 \mathrm{IC}_{50}$ of $7.24 \mathrm{nM}$ and a Peak $2 \mathrm{IC}_{50}$ of $5.73 \mathrm{nM}$. Sigmoidal least squares fit with hillslopes of 1.5 and 1.6 for resting- and inactivated-state curves, respectively. 


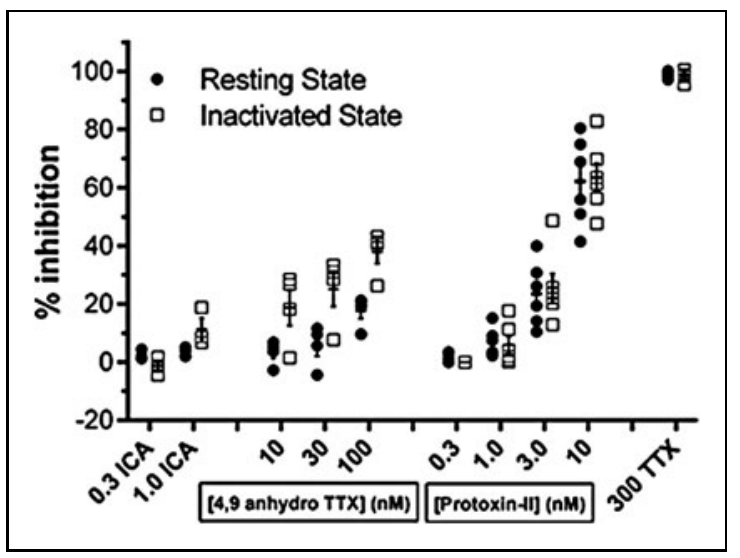

Fig. 9. Individual components of TTX-S current in ND7-23 cell inhibited by selective concentrations of Nav1.3, Nav1.6, and Nav1.7 antagonists. Individual cell and mean \% inhibition ( \pm SEM) of resting-state (filled circles) and inactivated-state TTX-S currents (open squares) in the presence of the indicated $\mu \mathrm{M}$ concentrations of the Nav1.3 inhibitor ICA $(N=5)$ and nanomolar concentrations of the Nav1.6 antagonist, 4,9 anhydro TTX $(N=4)$, and Nav1.7 blocker, Protoxin-II $(N=6)$, are shown from independent samples of $N D_{7-23}$ cells. Note the moderate inactivated state preference of the Icagen compound and 4,9 anhydro TTX compared with equipotent inhibition of resting- and inactivated-state TTX-S currents by ProtoxinII. A concentration of $300 \mathrm{nM} \mathrm{TTX}$ added to each cell at the end of every experiment completely reduced the remaining resting- and inactivated-state currents (resting state $98.5 \% \pm 0.64 \%$ of vehicle control amplitude $(N=26)$. ICA, Icagen compound 68 .

0.1\% DMSO vehicle (Fig. 7). A full four-point $\mathrm{IC}_{50}$ application protocol (0.3-10 nM in half log-unit increments) was employed to establish the potency of Protoxin-II in ND7-23 cells and determine the proportion of endogenous Nav current carried by Nav1.7 channels. There was no significant effect of 0.3 or $1 \mathrm{nM}$ Protoxin-II on inward currents, but higher concentrations of Protoxin-II that began to exceed the $\mathrm{IC}_{50}$ determined against hNav1.7 channels $(\sim 3.5 \mathrm{nM})$ produced significant inhibition of endogenous TTX-S currents in ND723 cells (Fig. 8C, D). Inhibition by 3 and $10 \mathrm{nM}$ Protoxin-II was relatively slow (Fig. $8 D$ ), and maximal block by the saturating, but selective, concentration of $10 \mathrm{nM}$ Protoxin-II ranged between $40 \%$ and $80 \%$ in individual cells. In contrast to the effects of 4,9 anhydro TTX, both resting-state Peak 1 (Fig. 8C) and half-inactivated-state Peak 2 currents (Fig. $8 D$ ) were equally sensitive to Protoxin-II, yielding $\mathrm{IC}_{50}$ values of 7.2 and $5.7 \mathrm{nM}$ against the resting-state Peak 1 and half-inactivatedstate Peak 2 currents, respectively (Fig. 8E).

The average effects on TTX-S currents of selective Nav1.3, Nav1.6, or Nav1.7 antagonists added alone to ND7-23 cells are summarized in Figure 9 and Table 1. An Nav1.3-selective concentration of $300 \mathrm{nM}$ of the Icagen compound 68 produced no effect on inward currents, while $1 \mu \mathrm{M}$ reduced resting-state

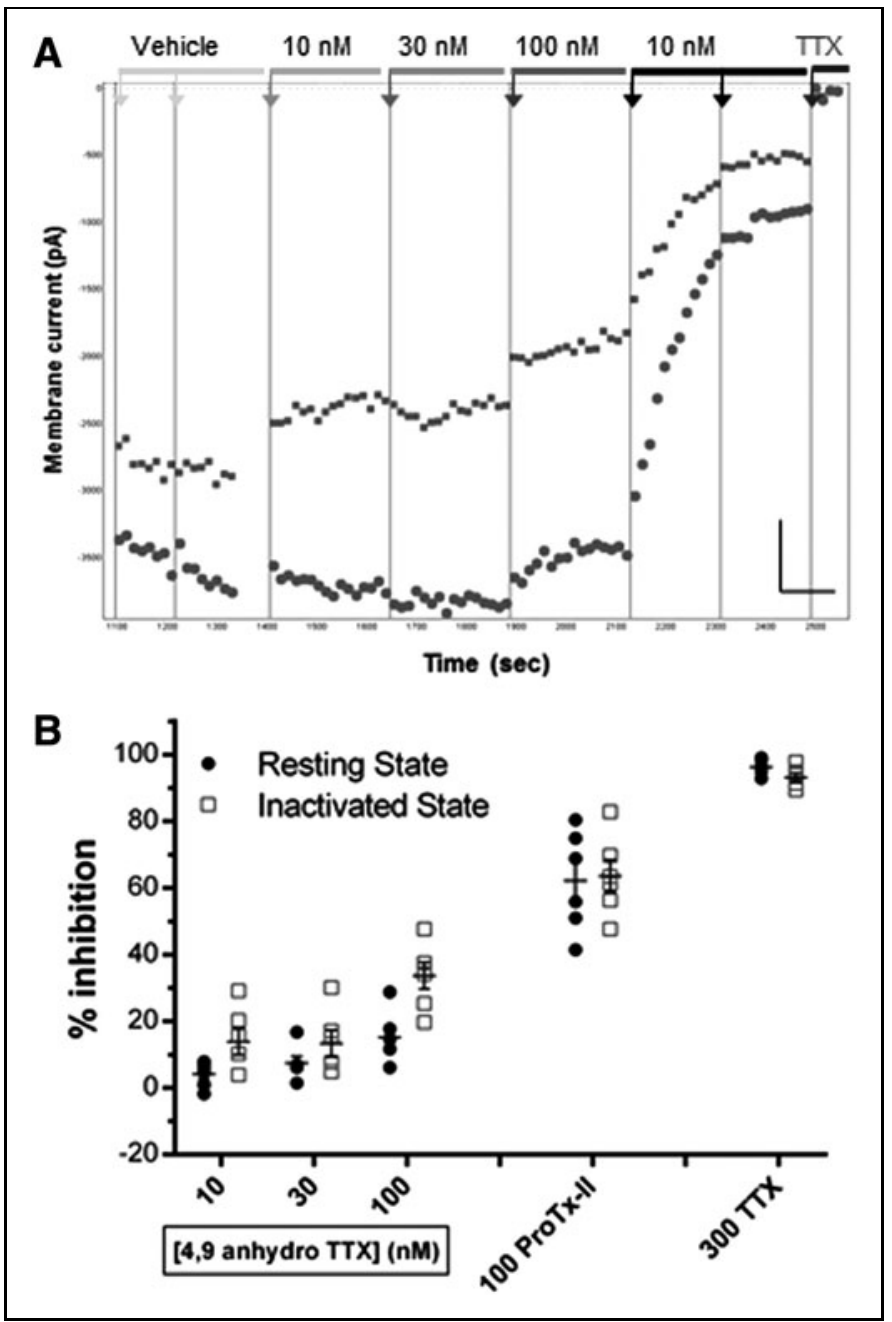

Fig. 10. Additive effects of selective Nav1.6 and Nav1.7 antagonists on TTX-S currents in ND7-23 cells. (A) Example IT plot of the additive effects of exposing an ND7-23 cell to Nav1.6-selective concentrations of 4,9 anhydro TTX (10-100 nM), followed by a saturating, but Nav1.7-selective, concentration of Protoxin-II (10 nM). Arrows indicate timing and concentration of compound applications. Restingstate (৩) and inactivated-state current amplitudes ( $\square$ ) are stable in vehicle control (0.05\% BSA), moderately inhibited by $100 \mathrm{nM} \mathrm{4,9}$ anhydro TTX, but slowly and almost completely reduced by $10 \mathrm{nM}$ Protoxin-II; all remaining inward currents are blocked by $300 \mathrm{nM}$ TTX. Scale bar is $500 \mathrm{pA}$ on the $y$-axis and $100 \mathrm{~s}$ on the $\mathrm{x}$-axis. (B) Summary \% inhibition data from $N=6$ individual cells (mean \pm SEM shown by bars) for resting-state (filled circles) and inactivated-state currents (open squares) after additive application of selective concentrations of 4,9 anhydro TTX and Protoxin-II to each cell, followed by $300 \mathrm{nM}$ TTX (which inhibited resting-state currents to $97.2 \% \pm 0.6 \%$ of vehicle control amplitude).

Peak 1 current by $0.7 \% \pm 3.0 \%$ and inactivated Peak 2 current by $8.5 \% \pm 1.7 \%$ (mean \pm SEM, $N=5$ ). However, as this concentration was nearly $20 \times$ higher than the heterologous Nav1.3 Peak 2 IC $_{50}$, these values should be considered an upper estimate on the contribution of native Nav1.3 channels to TTX-S 
currents in ND7-23 cells. The Nav1.6 inhibitor 4,9 anhydro TTX produced moderate, but dose-dependent, effects on TTX-S currents, reducing resting-state Peak 1 current by 3.3\% $\pm 2.1 \%$ at $10 \mathrm{nM}, 5.6 \% \pm 3.6 \%$ at $30 \mathrm{nM}$ and by $17.8 \% \pm 2.8 \%$ at $100 \mathrm{nM}$ (mean $\pm \mathrm{SEM}, N=4$ ). As noted above and by Rosker et al., ${ }^{30}$ this antagonist had greater potency against the inactivated state of Nav1.6 channels, with Peak 2 currents in ND7-23 cells inhibited by $18.7 \% \pm 6.2 \%, 25.1 \% \pm 5.9 \%$, and $37.9 \% \pm 3.9 \%$ in the presence of 10, 30, and 100 nM 4,9 anhydro TTX, respectively. In contrast, Protoxin-II reduced the amplitude of resting- and inactivated-state TTX-S currents equally well, with minor effects at $1 \mathrm{nM}$ (average of 5.6\% \pm 3.6\% inhibition of Peak 1 and $6.0 \% \pm 7.0 \%$ inhibition of Peak 2 , respectively; mean \pm SEM from $N=6$ cells) increasing to $22.4 \% \pm 10.5 \%$ and $25.5 \% \pm 12.1 \%$ at $3 \mathrm{nM}$, and reaching a maximum inhibition of $62.2 \% \pm 14.9 \%$ for Peak 1 resting-state currents and $63.6 \% \pm 12.0 \%$ of Peak 2 half-inactivated currents at $10 \mathrm{nM}$ (Fig. 9 and Table 1). Summing the effects of maximal, but selective, concentrations of Nav1.3, Nav1.6, and Nav1.7 inhibitors added individually to single ND7-23 cells reveals an overall reduction of resting TTX-S currents of $80.2 \%$ and complete (100.9\%) inhibition of inactivated-state currents.

Finally, we additively applied selective concentrations of 4,9 anhydro TTX and Protoxin-II to the same ND7-23 cells to assess the variation in contribution of Nav1.6 and Nav1.7 channels to endogenous TTX-S currents in single cells. Figure $10 \mathrm{~A}$ illustrates the result for an exemplar cell, showing the minor, but discernibly, dose-dependent inhibition of both resting-state and half-inactivated state currents with ascending doses of 4,9 anhydro TTX (10-100 nM), followed by slow, but profound, inhibition of the majority of the remaining endogenous Nav current by $10 \mathrm{nM}$ Protoxin-II. Addition of a saturating dose of $300 \mathrm{nM}$ TTX at the end of the experiment reduced peak inward currents to $<5 \%$ of initial values, consistent with the fact that essentially all of the endogenous currents in ND7-23 cells are carried by TTX-S channels. The average data from these experiments are shown in Figure $10 B$, showing that a minor component ( $20 \%)$ of total inward current is inhibited by a concentration of $100 \mathrm{nM}$ 4,9 anhydro TTX that is selective for Nav1.6 channels. The majority of the remaining TTX-S currents in these cells are blocked by a concentration of Protoxin-II (10 nM) selective for Nav1.7 channels, reducing total peak inward current by $73.7 \% \pm 3.5 \%$ compared with the vehicle period (mean \pm SEM from $N=6$ cells). As noted above (Fig. 9), the inhibition of inactivated-state Peak 2 currents by 100 nM 4,9 anhydro TTX is somewhat greater than its inhibition of resting-state Peak 1 currents, leading to a small increase in the overall proportion of endogenous inactivated-state TTX-S current that is blocked after the additive exposure of single ND7-23 cells to 4,9 anhydro TTX and Protoxin-II (82.1\% $\pm 2.7 \%$, mean \pm SEM from $N=6$ cells). Thus, we conclude that $\sim 75 \%-80 \%$ of the total inward TTX-S currents in ND7-23 cells are carried by a combination of Nav1.7 > Nav1.6 channels.

\section{DISCUSSION}

Our first significant finding was to confirm the potency and selectivity of 4,9 anhydro TTX for human Nav1.6 channels that were originally demonstrated using rodent channels ${ }^{30,42}$ and to demonstrate its utility as a subtype-selective ligand for studying the function of rodent and human Nav1.6 channels implicated in pain signaling, epilepsy, and multiple sclerosis. Our second major finding was that functional patch clamp electrophysiology largely confirms previous molecular profiling to reveal that $75 \%-80 \%$ of the endogenous inward sodium currents in ND7-23 cells are carried by Nav1.7 > Nav1.6 >> Nav1.3 channels. We also confirmed previous findings that $>95 \%$ of the total Nav currents in these cells were due to activity of TTX$S$ channels. The remaining small fraction $(\sim 20 \%)$ of TTX-S currents could not be accurately assigned to a particular Nav1. $\mathrm{X}$ subtype owing to limits in the selectivity of the pharmacological ligands available. Finally, we demonstrate that native ND723 neuronal cells display very stable endogenous TTX-S Nav currents in whole-cell recordings and are amenable for automated patch clamping on robot platforms normally employed for ion channel screening. This combination of physiological and biophysical features makes ND7-23 cells a useful native neuronal system suitable for the Nav1.x gene family and species selectivity testing in ion channel drug discovery screening cascades as well as translational phenotypic drug discovery assays.

Selectivity for Human Nav1.6 Channels and Mechanism of Action of 4,9 Anhydro TTX

When designing these experiments, we were surprised that the initial finding of the Nav1.6 potency and selectivity of 4,9 anhydro $\mathrm{TTX}^{30}$ had not been extended to human Nav1.X channels expressed in a mammalian cell background, although moderate potency and markedly reduced selectivity had been shown in rat Nav1.6 and human Nav1.2 channels expressed in HEK cells. ${ }^{42}$ Nav1.6 is an important target for the regulation of axonal excitability ${ }^{40}$ and pain transmission ${ }^{47}$ and is implicated in diseases such as multiple sclerosis and epilepsy, ${ }^{11,41}$ so a ligand with a reported 150-fold selectivity for Nav1.6 should be attractive for drug discovery purposes. We therefore set out to confirm data from the previous studies (which predominantly used rodent Nav1.x channels) using human Nav1.x channels expressed in mammalian cells before using 4,9 anhydro TTX as 
a selective ligand to reveal the repertoire of endogenous TTX-S channels in ND7-23 cells.

We determined the potency and selectivity of 4,9 anhydro TTX for heterologous hNav1.6 channels using the same QPatch APC platform and voltage protocols we would employ for Nav1.x profiling in ND7-23 cells. We confirmed many features of inhibition of Nav1.6 channels by 4,9 anhydro TTX originally described ${ }^{30}$ including the slightly slower block compared with TTX (Fig. 3C), low nanomolar potency (Fig. 3E), and additional effects on the inactivated state (Fig. 3C, E; also see Figs. 9 and 10). We also confirmed the significant selectivity of 4,9 anhydro TTX for Nav1.6 over Nav1.7 channels (Fig. 4) using human channels expressed in a mammalian cell background, although the 50 -fold selectivity ratio we calculated is lower than the $\sim 150$-fold ratio reported in oocytes, ${ }^{30}$ but higher than the 5 -fold selectivity for Nav1.x channels expressed in HEK cells. ${ }^{42}$ The QPatch $\mathrm{IC}_{50}$ for inhibition of resting-state hNav1.6 Peak 1 currents $(32.9 \mathrm{nM})$ was fourfold less potent than that reported for mouse channels expressed in Xenopus oocytes, but fourfold lower than that against rat Nav 1.6 channels in HEK cells, ${ }^{42}$ which can be attributed to a number of experimental differences (e.g., solutions, whole-cell perfusion vs. intracellular impalement, compound flow rate, and volume) as well as biological, species, and expression system variables. However, the hNav1.7 potency of 4,9 anhydro TTX $(1.62 \mu \mathrm{M})$ obtained in mammalian cells on the QPatch is very close to that reported for hNav1.7 channels expressed in oocytes $(1.27 \mu \mathrm{M})$, suggesting that Nav1.6 species differences may be the most important issue for this ligand.

Species differences in the efficacy of 4,9 anhydro TTX may be hard to explain if one assumes it acts like its parent TTX as a simple pore blocker binding to a specific tyrosine residue in the S5-S6 pore loop of domain I. However, we have observed and others have published that TTX exhibits moderate use- and state-dependent inhibition of Nav channels, and the underlying mechanism of this effect is still being determined. ${ }^{43,44}$ We note that 4,9 anhydro TTX had greater potency against the inactivated state compared with the resting/open state of hNav1.6 channels in heterologous HEK cells (Fig. 3), as well as native TTX-S currents in ND7-23 cells (Figs. 9 and 10), indicative of some additional state-dependent mechanism of action. Similarly, although Rosker et al. ${ }^{30}$ noted that most of the effects of TTX and 4,9 anhydro TTX on Nav1.x channels were due to open-state tonic block, supplemental data showed that TTX and 4,9 anhydro TTX $(10 \mathrm{nM})$ produced a statistically significant leftward shift in the inactivation curve of mouse Nav1.6 channels. This would indicate that 4,9 anhydro TTX (and TTX) exhibits a more complex mechanism of action, which also involves a state-dependent preference for the inactivated conformation of Nav1.6 channels. This could involve binding to a more divergent set of amino acid residues of the Nav1.6 channel protein in or adjacent to the outer vestibule, enhancing the possibility of Nav1.6 subtype specificity, as well as species differences. Therefore, the lower potency and efficacy (i.e., partial block, Fig. 3) of 4,9 anhydro TTX against human Nav1.6 channels in this study compared with previous work on mouse channels may be explained by species differences in inactivated-state residues and channel conformations, leading to abrogation of an additional binding mechanism.

Repertoire of Functional Nav1.x Currents Endogenously Expressed in ND7-23 Cells

Nav1.3. There is a consistent pattern for very low expression of Nav1.3 channels in ND7-23 cells as evidenced by molecular, biophysical, and pharmacological methods. Polymerase chain reaction (PCR) transcripts are low, ${ }^{32,33}$ and usedependent block of TTX-S currents by ambroxol in ND7-23 cells is more similar to that seen with heterologous Nav1.7 than Nav1.3 channels. ${ }^{32}$ Our QPatch results are consistent with these observations as we found negligible effects of saturating concentrations of a selective Nav1.3 smallmolecule antagonist on whole-cell TTX-S currents in ND7-23 cells (Figs. 6 and 9). As the Nav1.3-selective Icagen compound 68 is strongly state dependent (Fig. 2), we based our analysis on its effects on inactivated Peak 2 current amplitude. Thus, a saturating, but selective, concentration of $300 \mathrm{nM}$ of the Icagen compound 68 ( 5-fold over the heterologous Nav1.3 Peak 2 IC $_{50}$ concentration of $56 \mathrm{nM}$ ) had no significant effect on inactivated-state Peak 2 TTX-S current amplitude in ND723 cells $(-0.5 \%)$, while a potentially less selective concentration of $1 \mu \mathrm{M}$ reduced ND7-23 inactivated-state current by only $8.5 \%$. We conclude that Nav1.3 channels contribute at most $\sim 5 \%$ of the macroscopic TTX-S current in ND7-23 cells, but in all likelihood, they represent an even smaller fraction.

Nav1.6. In two separate sets of experiments, we determined that Nav1.6 channels carry a minimum of $15 \%-20 \%$ of TTX-S current evoked in ND7-23 cells from a resting state (Figs. 7B, 9, and 10), and based on the fact that inactivated-state Peak 2 Nav currents exhibit a higher affinity for block by 4,9 anhydro TTX, we estimate that a maximum of $\sim 35 \%$ of the endogenous TTX-S current in ND7-23 cells flows through Nav1.6 channels. We used a maximal concentration of $100 \mathrm{nM} \mathrm{4,9}$ anhydro TTX to selectively block Nav1.6 channels without affecting Nav1.7 currents, similar to a previous study in HEK cells expressing rat and human Nav1.x channels. ${ }^{42}$ Although 4,9 anhydro TTX also blocks Nav1.3 channels with a potency of $341 \mathrm{nM}$ in oocytes, ${ }^{30}$ we think it is unlikely that any of the effects of 4,9 anhydro TTX attributed to inhibition of Nav1.6 
in this study are due to inhibition of Nav1.3 for two reasons. First, the selective Icagen Nav1.3 antagonist had no significant effect on macroscopic currents in ND7-23 cells at $300 \mathrm{nM}$, five times its $\mathrm{IC}_{50}$ for heterologous Nav1.3 channels. Second, both Leffler et $a l^{32}$ and Kennedy et al. $^{33}$ reported seeing low or barely detectable levels of Nav1.3 transcript expression in ND7-23 cells. Thus, we conclude that the previous molecular and our pharmacological data suggest that there is negligible expression of functional Nav1.3 channels in ND7-23 cells and that the effects of moderate concentrations of 4,9 anhydro TTX (up to $100 \mathrm{nM}$ ) can be attributed to selective inhibition of Nav1.6 channels.

The intermediate level of Nav1.6 channel function that we detected is consistent with the PCR study of Kennedy et al., ${ }^{33}$ but contradicts the negative result obtained by Leffler et $a l^{32}$ Unpublished western blots indicate the presence of Nav1.6 protein in ND7-23 cells, but the relative levels compared with other Nav1.x channels were not described. ${ }^{48}$ Nav1.6 channels can generate characteristic resurgent currents during repolarization of the membrane potential, ${ }^{42}$ but this diagnostic feature appears to depend on the presence of $\beta 4$ accessory subunits, ${ }^{3,49}$ which are lacking in ND7-23 cells, ${ }^{7}$ and there is no evidence for such a biophysical phenomenon in our QPatch recordings (data not shown). Nevertheless, the presence of functional Nav1.6 channels in ND7-23 cells, alongside Nav1.7 channels, makes them a useful model of sensory neuron action potential generation and axonal conduction for translational studies of pain signal transmission and drug discovery.

Nav1.7. The effects of Protoxin-II on TTX-S currents in ND7-23 were quite consistent, with application of cumulative concentrations in the $\mathrm{IC}_{50}$ experiments producing dose-dependent reductions in resting- and inactivated-state current amplitude that reached a maximum inhibition of 62\% (Figs. 8 and 9), while the net inhibition by a saturating dose of $10 \mathrm{nM}$ ProtoxinII (applied after $100 \mathrm{nM} 4,9$ anhydro TTX) was also 60\% (75\% total $-15 \%$ after 4,9 anhydro TTX, Fig. 10). As the QPatch IC $_{50}$ for Protoxin-II inhibition of heterologous hNav1.7 channels (3.6 nM) is very close to that for ND7-23 TTX-S currents (7.2 nM), and the top concentration of $10 \mathrm{nM}$ inhibits $>90 \%$ of macroscopic Nav1.7 currents without affecting other human Nav1.x channels, ${ }^{27}$ we are confident in our estimate that $60 \%$ of the TTX-S current in ND7-23 cells is carried by Nav1.7 channels. Our pharmacological data that Nav1.7 is the predominant functional TTX-S species in ND7-23 cells are also consistent with previous molecular studies, which detected Nav1.7 as the most abundant PCR transcript. ${ }^{32,33}$

Unlike the pore blocker TTX, Protoxin-II is a gating modifier that binds to voltage sensor paddles in several domains of the
Nav1.7 channel to retard voltage-dependent activation. ${ }^{28,50}$ Although the binding mechanism of Protoxin-II is state dependent, it does not necessarily elicit a preference for inhibiting resting-state or inactivated-state Nav1.7 currents, as reflected in the similar maximal inhibition values and $\mathrm{IC}_{50}$ potencies for hNav1.7 and ND7-23 TTX-S currents (Figs. 5, 8, 9, and 10). Protoxin-II has to access more distant and cryptic binding pockets within the channel than TTX and so the kinetics of block are relatively slow, as seen in our QPatch experiments (Figs. 5, 8, and 10). We failed to achieve complete block with the highest (selective) concentration of $10 \mathrm{nM}$ Protoxin-II tested, and our Nav1.7 IC $_{50}$ of $3 \mathrm{nM}$ was 10 -fold less potent than that reported using longer applications of larger volumes of peptide under manual patch clamp conditions, ${ }^{27}$ although it is identical to that recently reported by Amgen scientists using similar protocols on the PatchXpress APC platform. ${ }^{50}$ Subsaturating block of Nav1.7 currents by Protoxin-II has been observed by others ${ }^{51}$ and our QPatch data are very similar to the $3 \mathrm{nM} \mathrm{IC}_{50}$ and 80\% maximal block seen with $10 \mathrm{nM}$ Protoxin-II in manual patch experiments reported by Smith et al. ${ }^{46} \mathrm{We}$ expected slow kinetics of block from previous studies in oocytes and heterologous cells and, for this reason, designed the QPatch application protocol to use multiple additions during each 2min application of each concentration to try and saturate the system, but although the perfusion capabilities of the QPatch system are well suited to studies of small volumes of expensive reagents such as toxins, it will always be less efficacious than the open-ended perfusion properties of a manual patch clamp system. Nevertheless, our data prove that it is possible to work with potent and sticky peptide toxins on an APC system and obtain results that are comparable with the gold standard of manual patch clamp and preserve the characteristic binding properties and blocking mechanisms of such important ion channel ligands.

In summary, a significant amount, but not all, of the endogenous TTX-S currents in ND7-23 cells can be accounted for by the fractions inhibited in different cells by application of selective concentrations of each of the Nav1.x antagonists (Fig. 9). The Icagen compound ( $300 \mathrm{nM}$, Nav1.3) inhibited $\sim 0.5 \%$ of inward TTX-S currents, while 4,9 anhydro TTX (100 nM, Nav1.6) reduced resting-state current amplitude by $18 \%$ and Protoxin-II (10 nM, Nav1.7) led to a $62 \%$ reduction in Peak 1 current magnitude. Summing these independent Nav1.x subtype inhibitions yields an overall fraction of peak TTX-S currents of 80\%, while addition of the fractions of Peak 2 inactivated currents affected by the same three compounds yields an overall inhibition of exactly 100\% (the major difference stemming from the greater effect of 4,9 anhydro TTX on the inactivated state of heterologous and presumably also 
endogenous Nav1.6 TTX-S channels). Similarly, additive application of selective concentrations of $100 \mathrm{nM} 4,9$ anhydro TTX (Nav1.6), followed by $10 \mathrm{nM}$ Protoxin-II (Nav1.7) in the same ND7-23 cells (Fig. 10), inhibits 73.7\% $\pm 3.5 \%$ of total inward resting-state current and $82.1 \% \pm 2.7 \%$ of half-inactivated Peak 2 current (mean \pm SEM, $N=6$ ). Therefore, we can account for 75\%-100\% of total TTX-S current in ND7-23 cells through a combination of Nav1.7 > Nav1.6 > Nav1.3 channels.

Significantly, the repertoire and rank order of functional Nav1.x channels we identified in ND7-23 cells (Nav1.7 > Nav1.6) are similar to the major composition of TTX-S currents in small-diameter DRG neurons and the pharmacological sensitivity of action potentials in unmyelinated C-fibers of the rodent sciatic nerve. ${ }^{40,52}$ It should be noted that the third and minor component of adult rodent DRG TTX-S responses was attributed to Nav1.1 channels, not Nav1.3, as found by molecular and pharmacological studies in ND7-23 cells. The presence of functional Nav1.1 channels in ND7-23 cells remains to be determined with appropriately selective antagonists, but the possibility remains as the molecular data are equivocal with PCR experiments failing to detect Nav1.1 transcripts, ${ }^{32}$ but unpublished western blots indicating the presence of Nav1.1 protein in ND7-23 cells. ${ }^{48}$ It may be that ND7-23 cells represent a less mature phenotype and express low levels of the embryonic Nav1.3 channel rather than adult Nav1.1; this may also explain the lack of endogenous TTX-R Nav1.8 and Nav1.9 channels in ND7-23 cells. This DRG-like profile of TTX-S current expression suggests that the ND7-23 cell line is a useful reagent in the design of in vitro ion channel assays for pain drug discovery, including medium- and highthroughput high content electrophysiology screens on APC platforms. Having a native cell reagent that not only works so well on these platforms but also captures the physiology and biophysics of native rodent DRG neurons could enable the translational benefits of a phenotypic assay to be realized higher up a traditional ion channel screening cascade and therefore allow the testing of more compounds earlier in the drug discovery process. This could in turn highlight suitable scaffolds with efficacy in preclinical animal models of pain physiology, reducing animal use (NC3R) both through reduced need for testing in native neuronal cells and higher in vivo success rates.

\section{Remaining TTX-S Current}

It is difficult to unequivocally define the small fraction $(\sim 15 \%-25 \%)$ of macroscopic TTX-S current that remains in ND7-23 cells in the presence of selective concentration of Nav1.x subtype-specific ligands. The first issue to consider is the efficacy and selectivity of the pharmacological tools we employed. For example, the remaining TTX-S current may be carried by unblocked Nav1.6 channels as $100 \mathrm{nM} \mathrm{4,9}$ anhydro TTX does not completely inhibit these channels (Fig. 3). However, higher concentrations of 4,9 anhydro TTX could not be used as they begin to inhibit Nav1.7 channels (Fig. 4). Similarly, the top concentration of $10 \mathrm{nM}$ Protoxin-II chosen for this study did not completely block all Nav1.7 channels expressed in HEK cells (Fig. 5), and the rate of inhibition was relatively slow in the ND7-23 experiments (Figs. 8D and 10A), so we may have underestimated the full fraction of TTX-S current carried by Nav1.7 channels. Higher concentrations of Protoxin-II would presumably have faster onset and more complete block of TTXS currents, but would have lost selectivity against Nav1.6 channels and may also activate sustained Nav1.7 currents. $^{45}$

We also assume that the concentration-response curves for the selective antagonists we obtained against heterologous human Nav1.x channels in CHO and HEK cells can be translated to the same subtypes of rodent Nav1.x channels in ND723 cells. Although this is likely to be true for Protoxin-II, ${ }^{27,45}$ and our IC $_{50}$ value for 4,9 anhydro TTX was fairly close to that obtained against mouse Nav1.6 channels, ${ }^{30}$ it was fourfold lower than that reported for rat Nav1.6 channels expressed in HEK cells, ${ }^{42}$ although those authors also used $100 \mathrm{nM} 4,9$ anhydro TTX as the top concentration that maintained specificity for Nav1.6. We did not confirm the species selectivity of Icagen compound 68, but studies with the close analog ICA-121431 suggest that its potency and selectivity are maintained between human and mouse, but not rat, Nav1.x channels. ${ }^{36}$ In this case, the otherwise Nav1.3-selective ICA-121431 had low nanomolar potency against rat Nav1.7 channels, but if the same were true for Icagen compound 68, we would expect application of $300 \mathrm{nM}$ to inhibit $>50 \%$ of TTX-S current as the Nav1.7selective antagonist Protoxin-II affects rodent and human Nav1.7 channels equally, but this was not seen (Fig. 6). Thus, the first explanation for the residual TTX-S current in ND7-23 cells in the current experiments is that it is carried by Nav channels of the most common endogenous subtypes (Nav1.7 > Nav1.6) that remain unblocked in the presence of specific, but subsaturating, concentrations of their selective ligands, Protoxin-II $(10 \mathrm{nM})$ and 4,9 anhydro TTX (100 nM). Alternatively, the residual TTX-S current may be carried by rat rather than mouse Nav1.3 channels resistant to the effects of Icagen compound 68, 4,9 anhydro TTX, or Protoxin-II, but we regard this as unlikely given the extremely low levels of Nav1.3 expression detected in ND7-23 cells by molecular methods. ${ }^{32,33}$

Another explanation for the residual TTX-S current is that it is carried by Nav1.x channels other than Nav1.3, Nav1.6, or Nav1.7. We doubt it is Nav1.1 as PCR experiments do not detect any such transcripts, ${ }^{32}$ although another study suggests 
the presence of Nav1.1 protein in unpublished western blots from ND7-23 cells. ${ }^{48}$ Similarly, the Icagen compound ICA121431 is a close analog of Nav1.3 patent compound 68 and is an almost equipotent ( $\left.\sim 20 \mathrm{nM} \mathrm{IC}_{50}\right)$ antagonist of Nav1.3 and Nav1.1, ${ }^{36}$ but application of a saturating concentration of $300 \mathrm{nM}$ of Icagen compound 68 had negligible effects on resting- or inactivated-state TTX-S currents in ND7-23 cells (Fig. 6). In contrast, it is possible that Nav1.2 channels may underlie the remaining 20\% of macroscopic Nav activity in ND7-23 cells as moderate molecular signals for Nav1.2 channels were seen in ND7-23 cells using PCR ${ }^{32}$ and western blotting. ${ }^{48}$ However, the lack of any commercially available selective Nav1.2 ligands at the time of these experiments made their identification difficult from a functional perspective; it has also been claimed that the pattern of opioid modulation of endogenous TTX-S currents in ND7-23 cells suggests that no functional Nav1.2 channels are present. $^{32}$

\section{Endogenous TTX-S Channels in Other}

Neuroblastoma Cell Lines

ND7-23 neuroblastoma cells are one of several immortalized neuroectodermal cell lines to express endogenous Nav1.7 channels, but they have proven to be the most amenable to automated patch clamping. In contrast, the mouse NIE-115 mouse neuroblastoma cell line predominantly expresses Nav1.3 channels ${ }^{53}$ although previous biophysical articles claimed it was Nav1.2. ${ }^{46}$ Rat GH3 pituitary neuroendocrine cells express Nav1.1, Nav1.2, Nav1.3, and Nav1.6 channel transcripts, ${ }^{54,55}$ but their relative contribution to functional macroscopic currents in these cells remains to be determined. The F11 neuronal cell line (similar to ND7-23 cells being a hybrid between mouse N18TG-2 neuroblastoma and embryonic rat DRG neurons) expresses Nav1.2, Nav1.3, and Nav1.6 transcripts, but not Nav1.7 channels, based on the effects of scorpion toxins and unpublished PCR data. ${ }^{56}$ The NG108-15 cell line is another mouse N18TG-2 neuroblastoma hybrid (with rat glioma cells), which expresses endogenous calcium and sodium currents; although the former are well characterized, there is uncertainty about the underlying Nav channel subtypes. PCR failed to detect Nav1.7 transcripts in undifferentiated NG108-15 cells, ${ }^{34}$ but differentiation produces a marked increase in Nav current density associated with expression of Nav1.7 as detected by immunohistochemistry, real-time PCR, and western blotting. ${ }^{57,58}$ Finally, the SH-SY5Y neuroblastoma cell line has been used extensively in pain drug discovery owing to its human origin. Ionix relied on it for expression of Nav1.8 for their pain program, ${ }^{17}$ and several groups at the University of Queensland used various molecular and pharmacological techniques to establish that human SH-SY5Y cells express hNav1.7 > hNav1.2
$>$ hNav1.3 channels. ${ }^{34,59}$ They designed several Nav subtypespecific fluorescence screening assays, including one to detect novel Nav1.7-selective spider toxins as part of their pain drug discovery program. ${ }^{60,61}$

The lack of Nav1.7 channel expression at the molecular or functional level in various neuroectodermal cell lines is surprising given the original cloning of the hNE Nav1.7 gene from a neuroendocrine carcinoma ${ }^{62}$ and the fact that Nav1.7 is in the same duplicated TTX-S gene cluster as Nav1.1, Nav1.2, and Nav1.3, ${ }^{1}$ all of which are expressed in the various cell lines described above. A recent publication provides a possible explanation as it was reported that Nav1.7 gene products are regulated by a natural antisense transcript; this factor is found in the brain, spinal cord, and several subtypes of DRG neurons that do not express Nav1.7 channels, but is absent in SH-SY5Y cells that do express hNav1.7 channels. ${ }^{63}$ Thus, it may be that Nav1.7 gene expression and transcript levels are selectively downregulated in neuroectodermal cell lines that express this antisense factor or that the process of hybridization between different rodent species and neuroblastoma cell backgrounds has introduced or activated the Nav1.7 silencing factor that was previously absent or quiescent in rat DRG progenitor cells.

\section{Lack of Endogenous TTX-R Channels in ND7-23 Cells}

The complete lack of expression of endogenous Nav1.8 or Nav1.9 TTX-resistant channel transcripts or currents in ND7-23 cells was unexpected given their hybridization from rat DRG neurons. For example, Zhou et al. ${ }^{18}$ found that $98.8 \%$ of endogenous Nav currents were blocked by 300 nM TTX and our results are consistent with the application of $300 \mathrm{nM}$ TTX (a concentration selective for TTX-S channels ${ }^{7}$ ), which at the end of every QPatch experiment inhibited $>97 \%$ of macroscopic inward Nav current in all cells analyzed. ND7-23 cells can, however, heterologously express human (and rat) Nav1.8 channels ${ }^{7,18-22}$ and also the more difficult human Nav1.9 channels. ${ }^{23-25}$ Icagen recently showed that hNav1.9 channels can be expressed in HEK cells through the coexpression of two undefined (presumably human) $\beta$ subunits; ${ }^{64}$ as ND7-23 cells express both $\beta 1$ and $\beta 3$ accessory subunits, ${ }^{7}$ this could suggest that although the latter rodent $\beta$ subunits can support the insertion and function of human TTX-R channels, it is $\beta 2$ and/or $\beta 4$ subunits that are required for expression of recalcitrant rodent TTX-R channels. Similarly, other accessory proteins and modulatory pathways necessary for surface expression of functional rodent TTX-R channels may have been lost during the hybridization of rat DRG neurons with mouse neuroblastoma cells. For example, Annexin p11 is absent from ND7-23 cells, but present in SH-SY5Y cells, which can express rodent 
Nav1.8 channels, ${ }^{17}$ and coexpression of p11 enables the transient expression of rodent Nav1.8 in ND7-23 cells. ${ }^{7,15,22}$ The recent success in achieving low levels of transient ${ }^{24}$ and stable expression $^{23}$ of hNav1.9 in ND7-23 cells without transfection of additional subunits suggests that expression of human, but not rodent, Nav1.9 is less dependent on Annexin p11 than hNav1.8 expression. Finally, the lack of any endogenous TTX-R Nav1.8 or Nav1.9 currents or PCR transcripts in ND7-23 cells may indicate fundamental inactivation of the endogenous rodent Nav1.8 and Nav1.9 genes in this cell line, perhaps occurring through effects on the TTX-R gene cluster ${ }^{1}$ or reflecting an embryonic differentiation state (hence the low-level expression of Nav1.3 transcripts and channels). Application of new generation gene-editing techniques such as CRISPR-Cas9 to ND723 cells may be able to resurrect the expression of endogenous TTX-R channels and create a more complete in vitro model of rodent DRG neurons suitable for APC ion channel screening and pain drug development that will meet NC3R guidelines to reduce animal use.

\section{ACKNOWLEDGMENTS}

The authors gratefully acknowledge Louise Webdale and Toni Cooper for their cell culture expertise, and Richard Davies and Neil Bruce for assistance with setting up and optimizing the QPatch Nav1.x and ND7-23 automated patch clamp assays at Xention. Dr. Tony Rush provided insightful advice and helpful feedback on the manuscript. The authors would also like to acknowledge permissions and materials obtained from their colleagues, Dr. Takashi Sasamura and Mrs. Yoko Sekioka (nee Ono), and Mr. Ari Alexandrou and Dr. Eddy Stevens. This work was partially supported by the Slovenian Research Agency (Grant No. Z1-5458).

\section{DISCLOSURE STATEMENT}

M.R. and R.W.K. were employees at Xention Ltd., when this work was carried out and are now employees and shareholders in Metrion Biosciences Ltd. The authors declare that they do not have any conflicting interests concerning the present study. For clarity, they wish to highlight the fact that this work follows on from an EU-funded grant (MAREX) that all of the authors were participants in, but the experiments described here were not part of, or funded by, this collaboration.

\section{REFERENCES}

1. Catterall WA, Goldin AL, Waxman SG: International Union of Pharmacology. XLVII. Nomenclature and structure-function relationships of voltage-gated sodium channels. Pharmacol Rev 2005;57:397-409.

2. Rogers $M$, Tang L, Madge DJ, et al.: The role of sodium channels in neuropathic pain. Semin Cell Dev Biol 2006;17:571-581.
3. Eijkelkamp N, Linley JE, Baker MD, et al:: Neurological perspectives on voltagegated sodium channels. Brain 2012;135(Pt 9):2585-2612.

4. England $\mathrm{S}$, Rawson D: Isoform-selective voltage-gated $\mathrm{Na}(+)$ channel modulators as next-generation analgesics. Future Med Chem 2010;2:775-790.

5. Black JA, Waxman SG: Sodium channels and microglial function. Exp Neurol 2012;234:302-315.

6. Moran $\mathrm{O}$, Nizzari M, Conti F: Endogenous expression of the beta1A sodium channel subunit in HEK-293 cells. FEBS Lett 2000;573:132-144.

7. John $\mathrm{VH}$, Main MJ, Powell $\mathrm{AJ}$, et al:: Heterologous expression and functional analysis of rat Nav1.8 (SNS) voltage-gated sodium channels in the dorsal root ganglion neuroblastoma cell line ND7-23. Neuropharmacology 2004;46:425-438.

8. Cummins TR, Dib-Haij SD, Black JA, et al.: A novel persistent tetrodotoxinresistant sodium current in SNS-null and wild-type small primary sensory neurons. J Neurosci 1999;19:RC43.

9. He B, Soderland DM: Human embryonic kidney (HEK293) cells express endogenous voltage-gated sodium currents and Nav1.7 sodium channels. Neurosci Lett 2010:469:268-272.

10. Sharkey LM, Cheng $X$, Drews $V$, et al.: The ataxia3 mutation in the $\mathrm{N}$-terminal cytoplasmic domain of sodium channel Nav1.6 disrupts intracellular trafficking. J Neurosci 2009;29:2733-2741.

11. Oliva MK, McGarr TC, Beyer BJ, et al.: Physiological and genetic analysis of multiple sodium channel variants in a model of genetic absence epilepsy. Neurobiol Dis 2014;67:180-190.

12. Choi JS, Tyrell L, Waxman SG, et al:: Functional role of the C-terminus of voltage-gated sodium channel Nav1.8. FEBS Lett 2004;572:256-260.

13. Okuse $\mathrm{K}$, Malik-Hall M, Baker MD, et al:: Annexin II light chain regulates sensory neuron-specific sodium channel expression. Nature 2002;417:653-656.

14. Akiba I, Seki T, Mori M, et al:: Stable expression and characterization of human PN1 and PN3 sodium channels. Receptors Channels 2003:9:291-299.

15. CYL3025 PrecisIONTM: hNav1.8/B1-HEK Recombinant Stable Cell Line. www.merckmillipore.com/GB/en/product/PrecisION\%E2\%84\%A2-hNav1.8\%CE\% B21-HEK-Recombinant-Stable-Cell-Line,MM_NF-CYL3025. (Last accessed on [January 14, 2016]).

16. Berwick DC, Diss JKJ, Budhram-Mahadeo VS, et al.: A simple technique for the prediction of interacting proteins reveals a direct Brn-3a-androgen receptor interaction. J Biol Chem 2010;285:15286-15295.

17. Dekker LV, Daniels Z, Hick C, et al:: Analysis of human Nav1.8 expressed in SH-SY5Y neuroblastoma cells. Eur J Pharmacol 2005;528:52-58.

18. Zhou $X$, Dong XW, Crona J, et al.: Vinpocetine is a potent blocker of rat NaV1.8 tetrodotoxin-resistant sodium channels. J Pharmacol Exp Ther 2003;306:498-504.

19. Zhou $X$, Dong XW, Priestley T: The neuroleptic drug, fluphenazine, blocks neuronal voltage-gated sodium channels. Brain Res 2006;1106:72-81.

20. Dong XW, Goregoaker $S$, Engler $H_{1}$ et al:: Small interfering RNA-mediated selective knockdown of Nav1.8 tetrodotoxin-resistant sodium channel reverses mechanical allodynia in neuropathic rats. Neuroscience 2007;146:812-821.

21. Herold KF, Nau C, Ouyang W, et al:: Isoflurane inhibits the tetrodotoxin-resistant voltage-gated sodium channel Nav1.8. Anesthesiology 2009;111:591-599.

22. Yamaoka $K_{1}$ Inoue $M$, Miyazaki $K_{\text {, et }}$ al: Synthetic ciguatoxins selectively activate Nav1.8-derived chimeric sodium channels expressed in HEK293 cells. J Biol Chem 2009;284:7597-7605.

23. Vanoye CG, Kunic JD, Ehring GR, et al.; Mechanism of sodium channel NaV1.9 potentiation by G-protein signalling. J Gen Physiol 2013;141:192-202.

24. Leipold E, Liebmann L, Korenke GC, et al:: A de novo gain-of-function mutation in SCN11A causes loss of pain perception. Nat Genet 2013;45:1399-1404.

25. Goral RO, Leipold E, Nematian-Ardestani E, et al:: Heterologous expression of Nav1.9 chimeras in various cell systems. Pflugers Arch 2015;467:2423-2435.

26. Gonzalez J, Termin A, Martinborough E, et al:: Compositions useful as inhibitors of voltage-gated sodium channels. 2006, US20060025415 A1.

27. Schmalhofer WA, Calhoun J, Burrows $R$, et al.: ProTx-II, a selective inhibitor of NaV1.7 sodium channels, blocks action potential propagation in nociceptors. Mol Pharmacol 2008;74:1476-1484.

28. Bosmans F, Swartz KJ: Targeting voltage sensors in sodium channels with spider toxins. Trends Pharmacol Sci 2010;31:175-182. 
29. Teramoto N, Yotsu-Yamashita $M$ : Selective blocking effects of 4,9anhydrotetrodotoxin, purified from a crude mixture of tetrodotoxin analogues, on Nav1.6 channels and its chemical aspects. Mar Drugs 2015;13:984-995.

30. Rosker $C$, Lohberger $B$, Hofer $D$, et al:: The $\Pi X$ metabolite 4,9-anhydro- $\Pi X$ is a highly specific blocker of the Nav1.6 voltage-dependent sodium channel. Am J Physiol Cell Physiol 2007;293:C783-C789.

31. Wood JN, Bevan SJ, Coote PR, et al:: Novel cell lines display properties of nociceptive sensory neurons. Proc Biol Sci 1990;241:187-194.

32. Leffler A, Reckzeh J, Nau C: Block of sensory neuronal Na+ channels by the secreolytic ambroxol is associated with an interaction with local anesthetic binding sites. Eur J Pharmacol 2010;630:19-28.

33. Kennedy PG, Montague $P$, Scott $F$, et al.: Varicella-zoster viruses associated with post-herpetic neuralgia induce sodium current density increases in the ND7-23 Nav-1.8 neuroblastoma cell line. PLoS One 2013;8:e51570.

34. Vetter I, Mozar CA, Durek T, et al.: Characterization of Nav types endogenously expressed in human SH-SY5Y neuroblastoma cells. Biochem Pharmacol 2012;83:1562-1571.

35. Bosmans F, Rash L, Zhu S, et al:: Four novel tarantula toxins as selective modulators of voltage-gated sodium channel subtypes. Mol Pharmacol 2006;69:419-429.

36. McCormack K, Santos S, Chapman ML, et al.: Voltage sensor interaction site for selective small molecule inhibitors of voltage-gated sodium channels. Proc Natl Acad Sci U S A 2013;110:E2724-E2732.

37. MAREX: Exploring Marine Resources for Bioactive Compounds: From Discovery to Sustainable Production and Industrial Applications 2010-2014. www.marex.fi/ (Last accessed on March 11, 2016).

38. Zidar N, Tomasic T, Kirby R, et al:: New substituted aminobenzenesulphonamides as modulators of voltage-gated sodium channels. Poster H013 at XXIII International Symposium on Medicinal Chemistry, Lisbon, Spain, 2014, p159. www.wiley-vch.de/vch/journals/2452/not2wis/4_ISMC_2014_posters.pdf (Last accessed March 11, 2016).

39. Zidar N, Tomasic T, Peigneur $S$, et al:: New isoform selective voltage-gated sodium channel modulators. Poster \#47 at IXth Joint Meeting in Medicinal Chemistry, Athens, Greece, June 7-10, 2015.

40. Wilson MJ, Yoshikami D, Azam L, et al:: $\mu$-Conotoxins that differentially block sodium channels Nav1.1 through 1.8 identify those responsible for action potentials in sciatic nerve. Proc Natl Acad Sci U S A 2011;108:10302-10307.

41. Craner MJ, Newcombe J, Black JA, et al:: Molecular changes in neurons in multiple sclerosis: altered axonal expression of Nav1.2 and Nav1.6 sodium channels and $\mathrm{Na}+/ \mathrm{Ca} 2+$ exchanger. Proc Natl Acad Sci U S A 2004;101:8163-8173.

42. Hargus NJ, Nigam A, Bertram EH III, et al.: Evidence for a role of Nav1.6 in facilitating increases in neuronal hyperexcitability during epileptogenesis. J Neurophysiol 2013;110:1144-1157.

43. Conti $F$, Gheri $A$, Pusch $M$, et al:: Use dependence of tetrodotoxin block of sodium channels: a revival of the trapped-ion mechanism. Biophys $J$ 1996; 71:1295:1312.

44. Huang CJ, Schild L, Moczydlowski EG: Use-dependent block of the voltagegated $\mathrm{Na}+$ channel by tetrodotoxin and saxitoxin: effect of pore mutations that change ionic selectivity. J Gen Physiol 2012;140:435-454.

45. Xiao Y, Blumenthal K, Jackson JO II, et al.; The tarantula toxins ProTx-II and Huwentoxin-IV differentially interact with human Nav1.7 voltage sensors to inhibit channel activation and inactivation. Mol Pharmacol 2010;78:11241134.

46. Smith JJ, Cummins TR, Alphy $\mathrm{S}$, et al.: Molecular interactions of the gating modifier toxin ProTx-II with NaV 1.5: implied existence of a novel toxin binding site coupled to activation. J Biol Chem 2007;282:12687-12697.

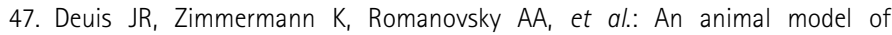
oxaliplatin-induced cold allodynia reveals a crucial role for Nav1.6 in peripheral pain pathways. Pain 2013;154:1749-1757.

48. Rusinova R, Herold KF, Sanford RL, et al.: Thiazolidinedione insulin sensitizers alter lipid bilayer properties and voltage-dependent sodium channel function: implications for drug discovery. J Gen Physiol 2011;138:249-270.
49. Tan ZY, Piekarz AD, Priest BT, et al.: Tetrodotoxin-resistant sodium channels in sensory neurons generate slow resurgent currents that are enhanced by inflammatory mediators. J Neurosci 2014;3:7190-7197.

50. Murray JK, Ligutti J, Liu D, et al.: Engineering potent and selective analogues of GpTx-1, a tarantula venom peptide antagonist of the NaV1.7 sodium channel. $J$ Med Chem 2015;58:2299-2314.

51. Middleton $R E$, Warren VA, Kraus $R L$, et al:: Two tarantula peptides inhibit activation of multiple sodium channels. Biochemistry 2002;41:14734-14747.

52. Zhang MM, Wilson MJ, Gajewiak J, et al:: Pharmacological fractionation of tetrodotoxin-sensitive sodium currents in rat dorsal root ganglion neurons by $\mu$-conotoxins. Br J Pharmacol 2013;169:102-114.

53. Jo S, Bean BP: Inhibition of neuronal voltage-gated sodium channels by brilliant blue G. Mol Pharmacol 2011;80:247-257.

54. Vega AV, Espinosa JL, López-Dominguez AM, et al.; L-type calcium channel activation up-regulates the mRNAs for two different sodium channel alpha subunits (Nav1.2 and Nav1.3) in rat pituitary GH3 cells. Brain Res Mol Brain Res 2003:116:115-125.

55. Baroni D, Plcco C, Barbieri R, et al.: Antisense-mediated post-transcriptional silencing of SCN1B gene modulates sodium channel functional expression. Biol Cell 2014;106:13-29.

56. Abbas N, Gaudioso-Tyzra C, Bonnet C, et al:: The scorpion toxin Amm VIII induces pain hypersensitivity through gain-of-function of TTX-sensitive $\mathrm{Na}+$ channels. Pain 2013;154:1204-1215.

57. Kawaguchi $A$, Asano $H$, Matsushima $K$, et al:: Enhancement of sodium current in NG108-15 cells during neural differentiation is mainly due to an increase in NaV1.7 expression. Neurochem Res 2007;32:1469-1475.

58. Liu J, Tu H, Zhang D, et al.: Voltage-gated sodium channel expression and action potential generation in differentiated NG108-15 cells. BMC Neurosci 2012;13:129.

59. Park JH, Park SJ, Chung MK, et al:: High expression of large-conductance Ca2+activated $\mathrm{K}+$ channel in the CD133+ subpopulation of SH-SY5Y neuroblastoma cells. Biochem Biophys Res Commun 2010;396:637-642.

60. Klint JK, Chin YK, Mobli M: Rational engineering defines a molecular switch that is essential for activity of spider-venom peptides against the analgesics target Nav1.7. Mol Pharmacol 2015;88:1002-1010.

61. Cardoso, FC, Dekan Z, Rosengren KJ, et al.: Identification and characterization of ProTx-III [ $\mu$-TRTX-Tp1a], a new voltage-gated sodium channel inhibitor from venom of the tarantula Thrixopelma pruriens. Mol Pharmacol 2015;88: 291-303.

62. Klugbauer N, Lacinova L, Flockerzi $V$, et al:: Structure and functional expression of a new member of the tetrodotoxin-sensitive voltage-activated sodium channel family from human neuroendocrine cells. EMBO J 1995;14: 1084-1090.

63. Koenig J, Werdehausen R, Linley JE, et al.: Regulation of Nav1.7: a conserved SCN9A natural antisense transcript expressed in dorsal root ganglia. PLoS One 2015;10:e0128830.

64. Lin Z, Santos S, Padilla KM, et al:: Biophysical and pharmacological properties of human Nav1.9 stably expressed in HEK 293 cells. Poster 35.20/B31, Chicago, Society for Neuroscience, October 17, 2015.

Address correspondence to: Marc Rogers, $P h D$ Metrion Biosciences Ltd. Building B501

Babraham Research Campus Cambridge CB22 3AT United Kingdom

E-mail: marc.rogers@metrionbiosciences.com 
ROGERS ET AL.

\section{Abbreviations Used}

$\mathrm{APC}=$ automated patch clamp

ATR $=$ attenuated total reflectance

$\mathrm{BSA}=$ bovine serum albumin

Cas9 $=$ CRISPR-associated protein 9

$\mathrm{CHO}=$ Chinese hamster ovary

$\mathrm{Cm}=$ capacitance

CRISPR $=$ clustered regularly interspaced short palindromic repeat

$\mathrm{DMF}=$ dimethyl formamide

DMSO $=$ dimethyl sulfoxide

$\mathrm{DRG}=$ dorsal root ganglion

EDTA $=2,2^{\prime}, 2^{\prime \prime}, 2^{\prime \prime \prime}$-(ethane-1,2-diyldinitrilo) tetraacetic acid

EGTA = ethylene glycol-bis(2-aminoethylether)- $N_{1} N_{1} N^{\prime}, N^{\prime}$-tetraacetic acid

$\mathrm{ESI}=$ electrospray ionization

$\mathrm{FT}-\mathrm{IR}=$ Fourier transform infrared spectroscopy

HEK = human embryonic kidney

HEPES $=2$-[4-(2-hydroxyethyl)piperazin-1-yl]ethanesulfonic acid

$\mathrm{HPLC}=$ high-performance liquid chromatography

$\mathrm{IC}_{50}=50 \%$ inhibitory concentration

$\mathrm{ICA}=$ Icagen compound 68

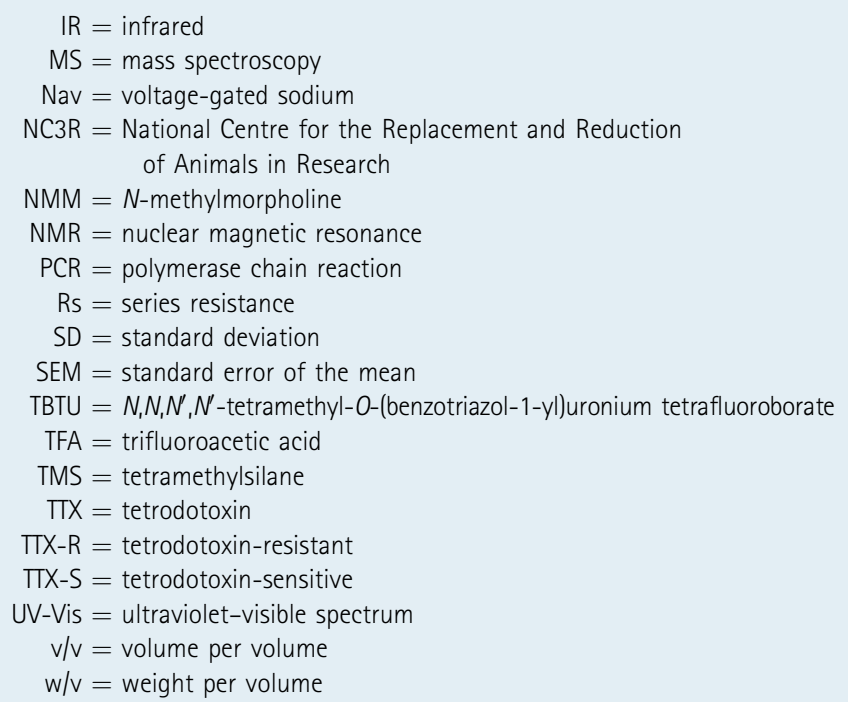

